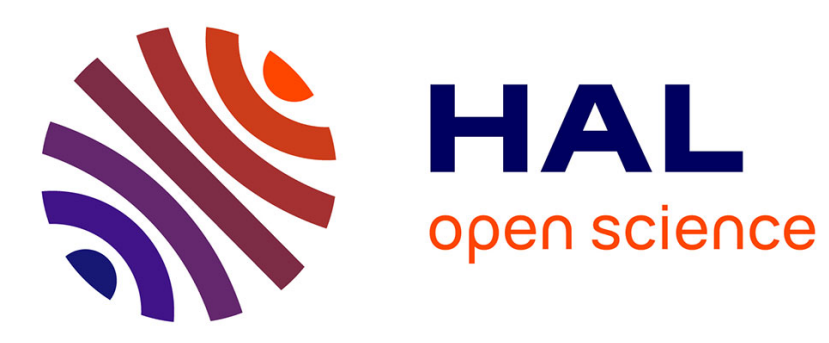

\title{
Hyperspectral Image Segmentation Using a New Spectral Unmixing-Based Binary Partition Tree Representation
}

Miguel Angel Veganzones, Guillaume Tochon, Mauro Dalla Mura, Antonio J. Plaza, Jocelyn Chanussot

\section{To cite this version:}

Miguel Angel Veganzones, Guillaume Tochon, Mauro Dalla Mura, Antonio J. Plaza, Jocelyn Chanussot. Hyperspectral Image Segmentation Using a New Spectral Unmixing-Based Binary Partition Tree Representation. IEEE Transactions on Image Processing, 2014, 23 (8), pp.3574 - 3589. 10.1109/TIP.2014.2329767 . hal-01010430

\author{
HAL Id: hal-01010430 \\ https://hal.science/hal-01010430
}

Submitted on 25 Nov 2015

HAL is a multi-disciplinary open access archive for the deposit and dissemination of scientific research documents, whether they are published or not. The documents may come from teaching and research institutions in France or abroad, or from public or private research centers.
L'archive ouverte pluridisciplinaire HAL, est destinée au dépôt et à la diffusion de documents scientifiques de niveau recherche, publiés ou non, émanant des établissements d'enseignement et de recherche français ou étrangers, des laboratoires publics ou privés. 


\title{
Hyperspectral image segmentation using a new spectral unmixing-based binary partition tree representation
}

\author{
M.A. Veganzones, Member, IEEE, G. Tochon, M. Dalla Mura, Member, IEEE, A. Plaza, Senior \\ member, IEEE, and J. Chanussot, Fellow, IEEE
}

\begin{abstract}
The Binary Partition Tree (BPT) is a hierarchical region-based representation of an image in a tree structure. BPT allows users to explore the image at different segmentation scales. Often, the tree is pruned to get a more compact representation and so the remaining nodes conform an optimal partition for some given task. Here, we propose a novel BPT construction approach and pruning strategy for hyperspectral images based on spectral unmixing concepts. Linear Spectral Unmixing (LSU) consists of finding the spectral signatures of the materials present in the image (endmembers) and their fractional abundances within each pixel. The proposed methodology exploits the local unmixing of the regions to find the partition achieving a global minimum reconstruction error. Results are presented on real hyperspectral data sets with different contexts and resolutions.
\end{abstract}

Index Terms

Binary Partition Trees, Hyperspectral Images, Spectral Unmixing, Segmentation.

\section{INTRODUCTION}

Imaging spectroscopy [14] (a.k.a. hyperspectral imaging) is concerned with the measurement, analysis, and interpretation of spectra acquired from a given scene or object [29]. The new generation of hyperspectral sensors

M.A. Veganzones was supported by the Delegation Generale de l'Armement (DGA) under contract PDE2012600079.

Copyright (c) 2013 IEEE. Personal use of this material is permitted. However, permission to use this material for any other purposes must be obtained from the IEEE by sending a request to pubs-permissions@ieee.org.

M.A. Veganzones, G. Tochon and M. Dalla Mura are with the Department Image and Signal (DIS), GIPSA-lab, Grenoble-INP, F-38402 Saint Martin d'Heres Cedex, France. Phone: +33 (0)4 7682 \{6396 / 6214 / 6411\}, fax: +33 (0)4 7657 4790. (e-mail: miguel-angel.veganzones@ gipsalab.fr; guillaume.tochon@gipsa-lab.fr; mauro.dalla-mura@gipsa-lab.fr)

A.J. Plaza is with the Hyperspectral Computing Laboratory, University of Extremadura (UEX), Cáceres, Spain. Phone: +34 927257000 (Dial extension 51662 during voice message). (e-mail: aplaza@uex.es)

J. Chanussot is with the Department Image and Signal (DIS), GIPSA-lab, Grenoble-INP, F-38402 Saint Martin d'Heres Cedex, France; and, with the Faculty of Electrical and Computer Engineering, University of Iceland, Reykjavik, Iceland. Phone: +33 (0)4 76826273 , fax: +33 (0)4 7657 4790. (e-mail: jocelyn.chanussot@gipsa-lab.fr) 
will collect a huge amount of hyperspectral data in the next decade, and the fields of knowledge that will benefit from the potential applications of hyperspectral imaging are huge [13], [29].

Besides, the high spectral resolution and the increase of the spatial resolution due to modern hyperspectral sensors and new super-resolution techniques [1], pose new challenges that should be faced. Most of the traditional processing algorithms fail when the spectral resolution increases significantly [3]. For instance, conventional statistical learning becomes intractable with hyperspectral data because of the increased dimensionality [10]. Thus, there has been a big increase in the research efforts conducted to develop new computational methods adapted to the specificities of hyperspectral data in order to analyse and fully exploit the information contained in hyperspectral images.

For instance, over the last few years, many efforts have been devoted to supervised classification of hyperspectral scenes [8], [11], [21], [29], [44]. In turn, unsupervised hyperspectral image segmentation has received comparatively much less attention. However, due to the limited amount of reference information generally available in practice, the unsupervised segmentation of hyperspectral images is an important area that still needs to be further developed. Unsupervised segmentation allows to explore and understand the contents of hyperspectral images without any $a$ priori knowledge.

Most available hyperspectral segmentation techniques are focused on analyzing the data as a set of unordered pixels, where the clustering process groups the data by means of their spectral information only. In [5], authors propose a new substractive-clustering-based similarity segmentation and a novel cluster validation method using One-Class Support Vector Machine (OC-SVM). In [12] unsupervised hyperspectral image segmentation based on the phase-correlation measure of subsampled hyperspectral data, referred to as modified phase correlation, is presented. In [2], authors make use of a fuzzy C-means (FCM) clustering algorithm to obtain segmentation maps that are later used to improve the classification of hyperspectral images. Authors in [30] propose a fast search method for vector quantization based on the generalized Lloyd algorithm (GLA). These clustering approaches as well as the classical k-Means and related algorithms require the number of clusters as an input and do not take any spatial information into account.

There is a growing interest in the development of unsupervised techniques taking into account both spectral and spatial information to provide hyperspectral segmentation maps [29]. In [34], an extension of the Watershed segmentation technique to hyperspectral images is investigated. The resulting segmentation map is then used to improve the classification performances. However, if the goal is the unsupervised segmentation map itself, the use of the Watershed technique generally yields oversegmented maps. Hierarchical segmentation using trees provides a flexible approach to image interpretation. Authors in [4], [33], [35] make use of this approach for the segmentation and classification of hyperspectral images. In [33], the method is based on the construction of a minimum spanning forest (MSF) from region markers automatically defined from classification results. By assigning a class of each marker to all the pixels within the region grown from this marker, a spectral-spatial classification map is obtained. A similar approach is followed in [4], where the notion of stochastic MSF is introduced. In [35], the Hierarchical SEGmentation (HSEG) algorithm, which combines region object finding with region object clustering, produces a hierarchical set of image segmentations. Here, the use of markers allows the automated selection of a single 
segmentation level. However, these approaches make use of labelled data, which in hyperspectral image analysis is a major issue as the availability of reliable ground-truth data is scarce and expensive to obtain.

Hierarchical segmentation algorithms have proved to be very valuable to explore and exploit the spatial content of images by providing a hierarchy of segmentations working at different scales. Recently, some authors have proposed the use of the Binary Partition Tree (BPT) to handle very high dimensional data such as hyperspectral data [36], [38], [39]. The BPT is a hierarchical region-based representation of an image in a tree structure [31]. In these works, two region models are commonly used for hyperspectral images: the first order parametric region model, which represents a region by its mean spectrum, and the non-parametric statistical region model, which models a region by its set of histograms (one histogram per spectral band). Both types of region models have their non-exhaustive associated family of merging criteria [38]. Often, the BPT is pruned to achieve a segmentation where the nodes of the pruned tree represent an optimal partition for some kind of application. Many pruning strategies have already been investigated in the BPT literature [36], [38], [39] to achieve a classical segmentation or to improve a further classification operation. However, none of these take full advantage of the high spectral information contained in hyperspectral images. In this work (which was sketched in [42]), we introduce for the first time in the literature the use of spectral unmixing for the construction and merging of a BPT hyperspectral representation and the posterior segmentation of the image.

A hyperspectral image is usually represented as the result of the linear combination of a set of spectral signatures corresponding to macroscopically pure materials (such as water, soil, etc...), named endmembers, weighted by a set of cover proportions comprised in a fractional abundance matrix. This is the so called Linear Mixing Model (LMM) [24]. The spectral unmixing process corresponds to the inverse problem: given an hyperspectral image, the goal is to find the endmembers and their per-pixel abundances. The conventional approach is to manually select the endmembers from a spectral library, or more often, automatically induce them from the image itself using an endmember induction algorithm (EIA) [7], [15], [19], [40]. The fractional abundances can then be estimated solving the equation systems of the LMM given the original image and the set of endmembers [22], [25]. In some works [17], [23], [28], the abundance maps can act as segmentation maps of their corresponding materials.

Spectral unmixing is one of the most important and widely used techniques in hyperspectral image analysis. Here, we provide a new framework for hyperspectral data interpretation that combines the benefits of both techniques, i.e., spectral unmixing and binary partition trees. This framework relies on a BPT representation based on the spectral and/or spatial information of the regions, that leads to merge regions with similar materials and/or in similar proportions; and, pruning strategies that yield to a segmentation map that is optimal in terms of spectral unmixing quality. The BPT representation allows one to exploit the increasing spatial information of modern hyperspectral imagery which, combined with the information provided by the spectral unmixing, makes the most of the spatialspectral variability contained in the hyperspectral images for their automatic segmentation. Our contribution is two-fold:

- We provide a new framework for hyperspectral data interpretation that combines spectral unmixing concepts with BPT representations. Specifically, we propose two pairs of region models and corresponding merging 
criteria, based on spectral unmixing concepts, that exploit the spectral mixture information in order to build a BPT representation of hyperspectral images. The first one defines a region model as the endmembers induced from the region by means of some EIA, and a merging criterion based on the spectral similarity between two regions. The second one introduces the fractional abundances of the induced endmembers, in addition to the spectral information provided by the aforementioned endmembers, in order to define a spectral-spatial region model. A spectral-spatial dissimilarity is used to define the corresponding merging criterion.

- We propose four novel pruning optimization criteria that produce a partition with minimum energy, being the energy of a partition defined as the combination of the average/maximum unmixing reconstruction error obtained from the partition and a regularization on the size/number of regions in the partition. The final result is an optimal segmentation of the hyperspectral scene in terms of spectral unmixing quality and segmentation complexity by means of a BPT representation, which represents an unique and innovative contribution in the field of hyperspectral data interpretation.

The remainder of the paper is organized as follows. The BPT construction and merging processes are explained in Section II. In Section III we outline some fundamental concepts about spectral unmixing. In Section IV we introduce the proposed segmentation methodology using a BPT representation and pruning by means of hyperspectral unmixing concepts. Then, we present the materials and experimental methodology in Section V, and we provide detailed results on two standard hyperspectral scenes: Pavia University and Cuprite, in Section VI and Section VII, respectively. Finally, we conclude the paper with some remarks and hints at potential future research lines in Section VIII.

\section{Binary PARtition TREes}

\section{A. Building a BPT}

In the BPT representation, the leaf nodes correspond to an initial partition of the image, which can be the individual pixels, or a coarser segmentation map. From this initial partition, an iterative bottom-up region merging algorithm is applied until only one region remains. This last region represents the whole image and corresponds to the root node. All the nodes between the leaves and the root result of the merging of two adjacent children regions. An example of BPT is displayed in Fig. 1. If the initial partition contains $n$ leaf nodes, the final BPT contains $2 n-1$ nodes.

Two notions are of prime importance when defining a BPT: i) the region model $\mathcal{M}_{\mathcal{R}}$ which specifies how a region $\mathcal{R}$ is modelled, and ii) the merging criterion $\mathcal{O}\left(\mathcal{M}_{\mathcal{R}_{\alpha}}, \mathcal{M}_{\mathcal{R}_{\beta}}\right)$, which is a distance measure between the region models of any two regions $\mathcal{R}_{\alpha}$ and $\mathcal{R}_{\beta}$. Each merging iteration involves the search of the two neighbouring regions which achieve the lowest pair-wise similarity among all the pairs of neighbouring regions in the current segmentation map. Those two regions are consequently merged.

Given a hyperspectral region $\mathcal{R}$, with $N_{\mathcal{R}}$ hyperspectral samples $\mathbf{r}_{j} \in \mathbb{R}^{q}, j \in 1 \ldots N_{\mathcal{R}}$, the first-order parametric model $\mathcal{M}_{\mathcal{R}}$ is defined by the average of the hyperspectral samples $\overline{\mathbf{r}}$ in each band $k=1, \ldots, q$ :

$$
\mathcal{M}_{\mathcal{R}}{ }^{(k)} \stackrel{d}{=} \bar{r}^{(k)}=\frac{1}{N_{\mathcal{R}}} \sum_{j=1}^{N_{\mathcal{R}}} r_{j}^{(k)} .
$$



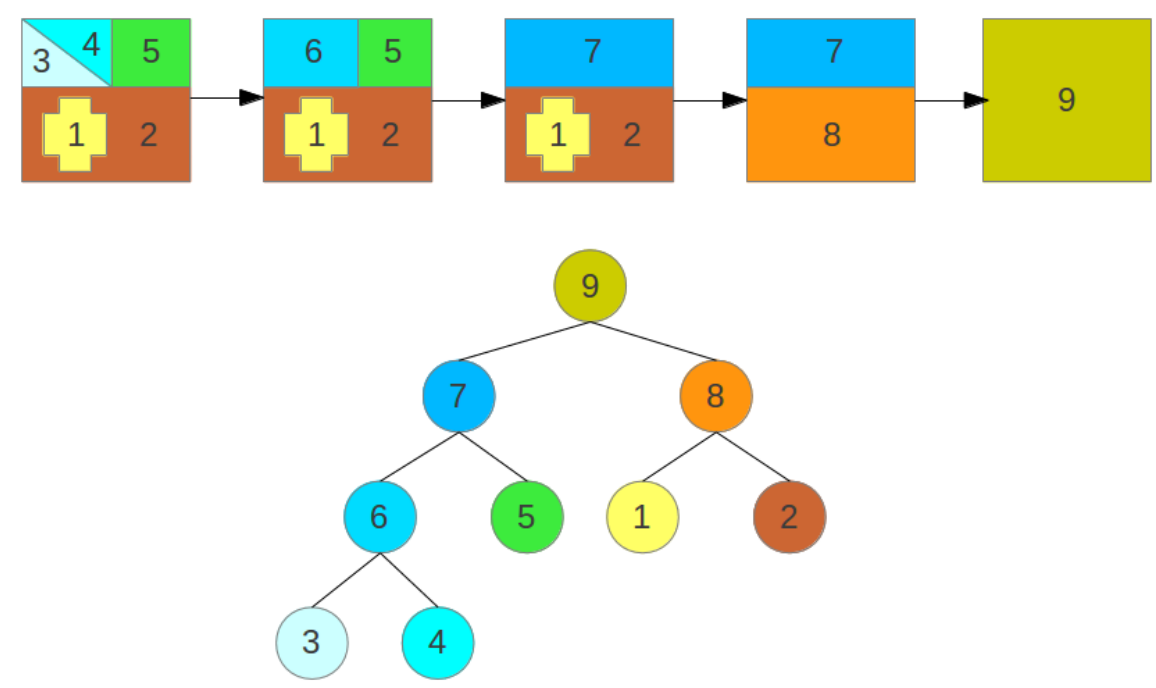

Fig. 1. Construction of the Binary Partition Tree (BPT).

Using the first-order parametric model (1), a merging criterion is defined as the spectral angle distance, $d_{\mathrm{SAD}}$, between the average values of any two adjacent regions:

$$
\mathcal{O}\left(\mathcal{M}_{\mathcal{R}_{\alpha}}, \mathcal{M}_{\mathcal{R}_{\beta}}\right) \stackrel{d}{=} d_{\mathrm{SAD}}\left(\overline{\mathbf{r}}_{\alpha}, \overline{\mathbf{r}}_{\beta}\right)=\arccos \left(\frac{\overline{\mathbf{r}}_{\alpha}^{T} \overline{\mathbf{r}}_{\beta}}{\left\|\overline{\mathbf{r}}_{\alpha}\right\|\left\|\overline{\mathbf{r}}_{\beta}\right\|}\right) .
$$

The building of a BPT may suffer from small and meaningless regions resulting in a spatially unbalanced tree. To overcome this limitation, a priority term is included in the merging criterion that forces those regions smaller than a given percentage of the average region size to be merged first [9], [36].

\section{B. Pruning a $B P T$}

The pruning step follows the construction of the BPT. If the construction of the BPT is generic once the region model and merging criterion have been defined, the pruning of the BPT is application dependant. Consequently, different pruning strategies are very likely to lead to different results. In the pruning operation, the branches of the tree are pruned such that the tree representation is reduced and the new leaves correspond to the regions achieving the most meaningful partition of the image with respect to the desired task.

There are two simple pruning strategies, the height-based pruning and the region-based pruning, that are often used to test that the BPT construction is reasonable and to serve as a baseline for the comparison with other pruning strategies. These two pruning strategies are application independent and thus, do not take full advantage of the BPT structure:

- The former consists in pruning the tree at some given height $h \geq 0$. The root is located at $h=0$ and its children are located at height $h=1$. Generalizing, the children from the nodes at height $h=i$ are located at height $h=i+1$. Fig. 2(a) shows an example of the use of the heigth-based pruning strategy to prune the aforementioned BPT (see Fig. 1) at heigth $h=1$. 
- The latter traverses the tree using an inverse order to its construction, pruning it once the number of regions in the partition reaches some given value $n_{r} \geq 0$. In other words, the final segmentation will be composed of the $n_{r}$ remaining regions before the completion of the tree. For instance, if the building of the nodes was done in the order $\mathcal{R}_{6} \rightarrow \mathcal{R}_{7} \rightarrow \mathcal{R}_{8} \rightarrow \mathcal{R}_{9}$ and $n_{r}=3$, the BPT will be pruned after the merging of regions $\mathcal{R}_{5}$ and $\mathcal{R}_{6}$ into $\mathcal{R}_{7}$ as it can be seen in Fig. 2(b).
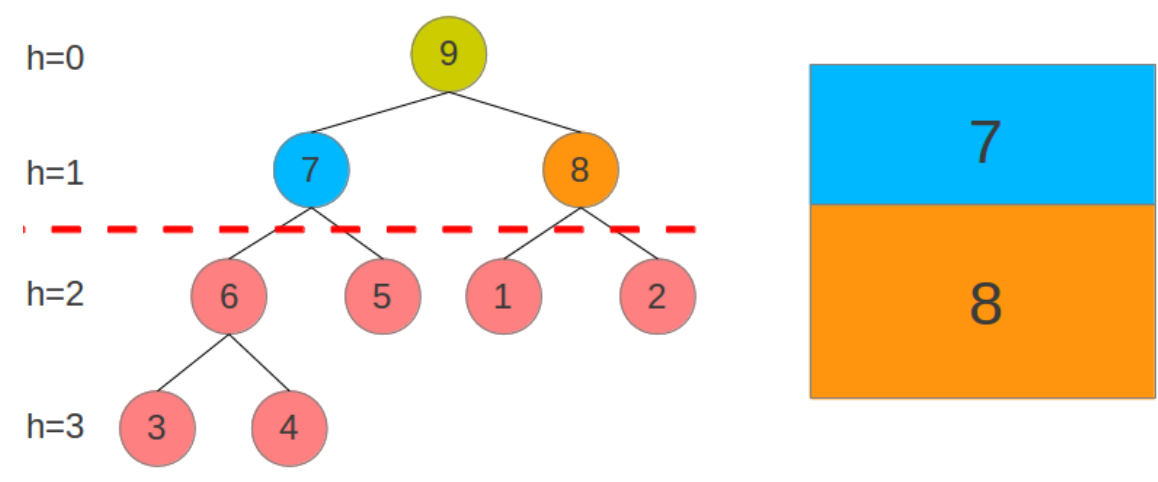

(a)

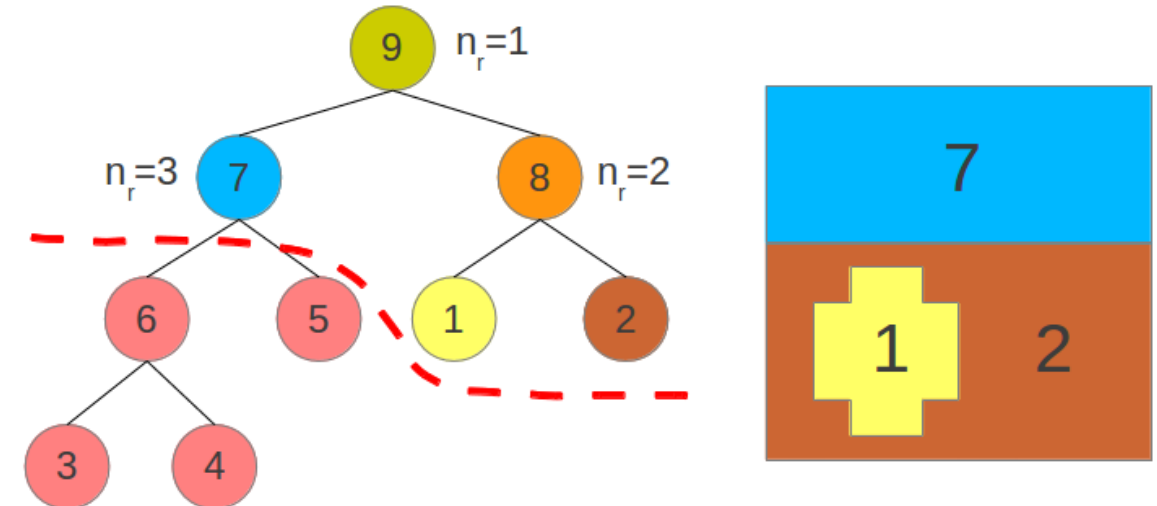

(b)

Fig. 2. (a) Height-based pruning of the Binary Partition Tree using $h=1$, and (b) Region-based pruning of the Binary Partition Tree using $n_{r}=3$.

In [37], the author proposes a pruning strategy to segment hyperspectral images based on the minimization of an energy function formulated as a Langrangian optimization problem:

$$
\mathcal{P}^{\star}=\arg \min _{\mathcal{P} \in \Omega} \sum_{\mathcal{R} \in \mathcal{P}}[D(\mathcal{R})+\lambda C(\mathcal{R})],
$$

where $\Omega$ denotes the set of all the partitions that could be obtained from the BPT representation of the image, $D(\mathcal{R})$ denotes a data fit error accounting for the region $\mathcal{R}$ in the partition, $C(\mathcal{R})$ denotes some complexity term, and $\lambda$ denotes the Lagrangian multiplier. In [37], author defines $D(\mathcal{R})$ as the Spectral Information Divergence 
(SID) measure according to the average spectrum of the region itself and its siblings:

$$
D(\mathcal{R})=\sum_{\mathbf{r} \in \mathcal{R}} \operatorname{SID}(\mathbf{r}, \overline{\mathbf{r}})+\sum_{\mathbf{r} \in \mathcal{R}_{l}} \operatorname{SID}\left(\mathbf{r}, \overline{\mathbf{r}}_{l}\right)+\sum_{\mathbf{r} \in \mathcal{R}_{r}} \operatorname{SID}\left(\mathbf{r}, \overline{\mathbf{r}}_{r}\right),
$$

where $\mathcal{R}_{l}$ and $\mathcal{R}_{r}$ denote the siblings of the region $\mathcal{R}, \overline{\mathbf{r}}$ denotes the average spectrum of a region, and:

$$
\operatorname{SID}(\mathbf{a}, \mathbf{b})=d_{\mathrm{KL}}(\mathbf{a}, \mathbf{b})+d_{\mathrm{KL}}(\mathbf{b}, \mathbf{a}),
$$

being $d_{\mathrm{KL}}(\cdot, \cdot)$ the Kullback Leibler divergence. The complexity term is defined as $C(\mathcal{R})=1$ aiming to minimize the number of regions in the partition. The author proposes in [37] a pruning strategy that looks for the optimum Lagrangian parameter, $\lambda^{*}$, that minimizes (3) subject to $\sum_{\mathcal{R} \in \mathcal{P}} C(\mathcal{R}) \approx C_{0}$, where $C_{0}$ denotes the number of regions in the partition (arbitrarily specified by the user).

\section{SPECTRAL UNMIXING}

Spectral unmixing pursues the decomposition of a hyperspectral image into the spectral signatures of its main constituents and their corresponding spatial fractional abundances. A common assumption in spectral unmixing is that the mixture of spectral signatures is linear. This yields to the LMM [7], [24] in which most of the unmixing methods are based on.

\section{A. Linear Mixing Model (LMM)}

The LMM states that a hyperspectral sample is formed by a linear combination of the spectral signatures of pure materials present in the sample (endmembers), plus some additive noise. Let $\mathbf{E}=\left[\mathbf{e}_{1}, \ldots, \mathbf{e}_{m}\right]$ be the pure endmember signatures (normally corresponding to macroscopic objects in the scene, such as water, soil, vegetation,...) where each $\mathbf{e}_{i} \in \mathbb{R}^{q}$ is a $q$-dimensional vector. Then, the hyperspectral signature $\mathbf{r}$ at each pixel in the image is defined by the expression:

$$
\mathbf{r}=\mathbf{s}+\mathbf{n}=\sum_{i=1}^{m} \mathbf{e}_{i} \phi_{i}+\mathbf{n},
$$

where $\mathbf{r}$ is given by the sum of the pixel's signal $\mathbf{s}$ and an independent additive noise component $\mathbf{n}$; and, $\phi$ is the $m$-dimensional vector of fractional per-pixel abundances subject to the Abundance Non-negative Constraint (ANC) and the Abundance Sum-to-one Constraint (ASC):

$$
\phi_{i} \geq 0 \forall i=1, \ldots, m \text { and } \sum_{i=1}^{m} \phi_{i}=1 .
$$

This equation can be extended to the whole image as $\mathbf{H}=\mathbf{E} \boldsymbol{\Phi}+\boldsymbol{\eta}$, where $\mathbf{H}$ denotes the hyperspectral image, $\boldsymbol{\Phi}$ is a matrix of fractional abundances and $\boldsymbol{\eta}$ is an independent additive noise.

\section{B. Endmember induction and abundance estimation}

Most of the times, the spectral signatures of the materials are unknown, and the set of endmembers must be built by either selecting spectral signatures from a spectral library, or by automatically inducing them from the 
image itself. Both can be performed manually or in an automatic way. In order to automatically induce the set of endmembers from the image, the use of some endmember induction algorithm (EIA) is required. The hyperspectral literature features plenty of such algorithms. Some reviews on the topic can be found in [7], [24], [40].

Once the set of endmembers, $\hat{\mathbf{E}}$, has been induced, their corresponding per-pixel abundances, $\hat{\mathbf{\Phi}}$, can be estimated by approximating a solution to an overdetermined linear system by the Least Squares method [25]. The FullyConstrained Least Squares Unmixing (FCSLU) method [22] solves the overdetermined linear system subject to ANC and ASC constraints.

The quality of the unmixing, $\hat{\mathbf{E}}$ and $\hat{\boldsymbol{\Phi}}$, at a given pixel $\mathbf{r}$ can be measured by the Root Mean Squared Error (RMSE) of the original hyperspectral signature with respect to the reconstructed one, $\hat{\mathbf{r}}=\sum_{i=1}^{m} \hat{\mathbf{e}}_{\mathbf{i}} \hat{\phi}_{i}$ :

$$
\epsilon(\mathbf{r}, \hat{\mathbf{r}})=\sqrt{\frac{1}{q} \sum_{k=1}^{q}\left(r^{(k)}-\hat{r}^{(k)}\right)^{2}} .
$$

\section{BPT CONSTRUCTION AND PRUNING BY MEANS OF HYPERSPECTRAL UNMIXING}

In this section, we introduce the adaptation of the BPT algorithm for hyperspectral unmixing purposes by defining a region model and merging criterion based on the induced endmembers and/or fractional abundances, and four pruning strategies based on the optimization of the spectral reconstruction error regularized by the segmentation complexity. Finally, we detail the novel methodology depicted in Fig. 3 to find an optimal segmentation of hyperspectral images from their BPT representation based on the information provided by the spectral unmixing.

\section{A. Novel unmixing-based region models and merging criteria}

We propose two novel region models and corresponding merging criteria based on spectral unmixing information extracted from the region. The first one is defined by means of the spectral information provided by the endmembers induced from the region. Thus we refer to this model as the spectral region model and merging criterion. In the second one, we propose to make use of the spatial information provided by the fractional abundances in addition to the corresponding endmembers. Therefore, we refer to this model as the spectral-spatial region model and merging criterion.

1) Spectral region model and merging criterion: For each region $\mathcal{R}_{i}$ a set of $m_{i}$ endmembers $\mathbf{E}_{\mathcal{R}_{i}}=\left[\mathbf{e}_{1}, \ldots, \mathbf{e}_{m_{i}}\right]$ is induced by an EIA, defining the spectral region model:

$$
\mathcal{M}_{\mathcal{R}_{i}} \stackrel{d}{=} \mathbf{E}_{\mathcal{R}_{i}}=\left[\mathbf{e}_{1}, \ldots, \mathbf{e}_{m_{i}}\right] .
$$

The spectral merging criterion between two regions $\mathcal{R}_{\alpha}$ and $\mathcal{R}_{\beta}$ modelled by (9) is given by the spectral dissimilarity between the set of endmembers of the two regions [16]:

$$
\mathcal{O}\left(\mathcal{M}_{\mathcal{R}_{\alpha}}, \mathcal{M}_{\mathcal{R}_{\beta}}\right) \stackrel{d}{=} s\left(\mathbf{E}_{\mathcal{R}_{\alpha}}, \mathbf{E}_{\mathcal{R}_{\beta}}\right)=\left\|\mathbf{m}_{r}\right\|+\left\|\mathbf{m}_{c}\right\|,
$$

where $\left\|\mathbf{m}_{r}\right\|$ and $\left\|\mathbf{m}_{c}\right\|$ are the minimum Euclidean norms among all row and column vectors, respectively, of the endmembers distance matrix $D_{\alpha, \beta}=\left[d_{k l}\right], k=1, \ldots, m_{\alpha}, l=1, \ldots, m_{\beta}$; being $d_{k l}$ the spectral angle distance 


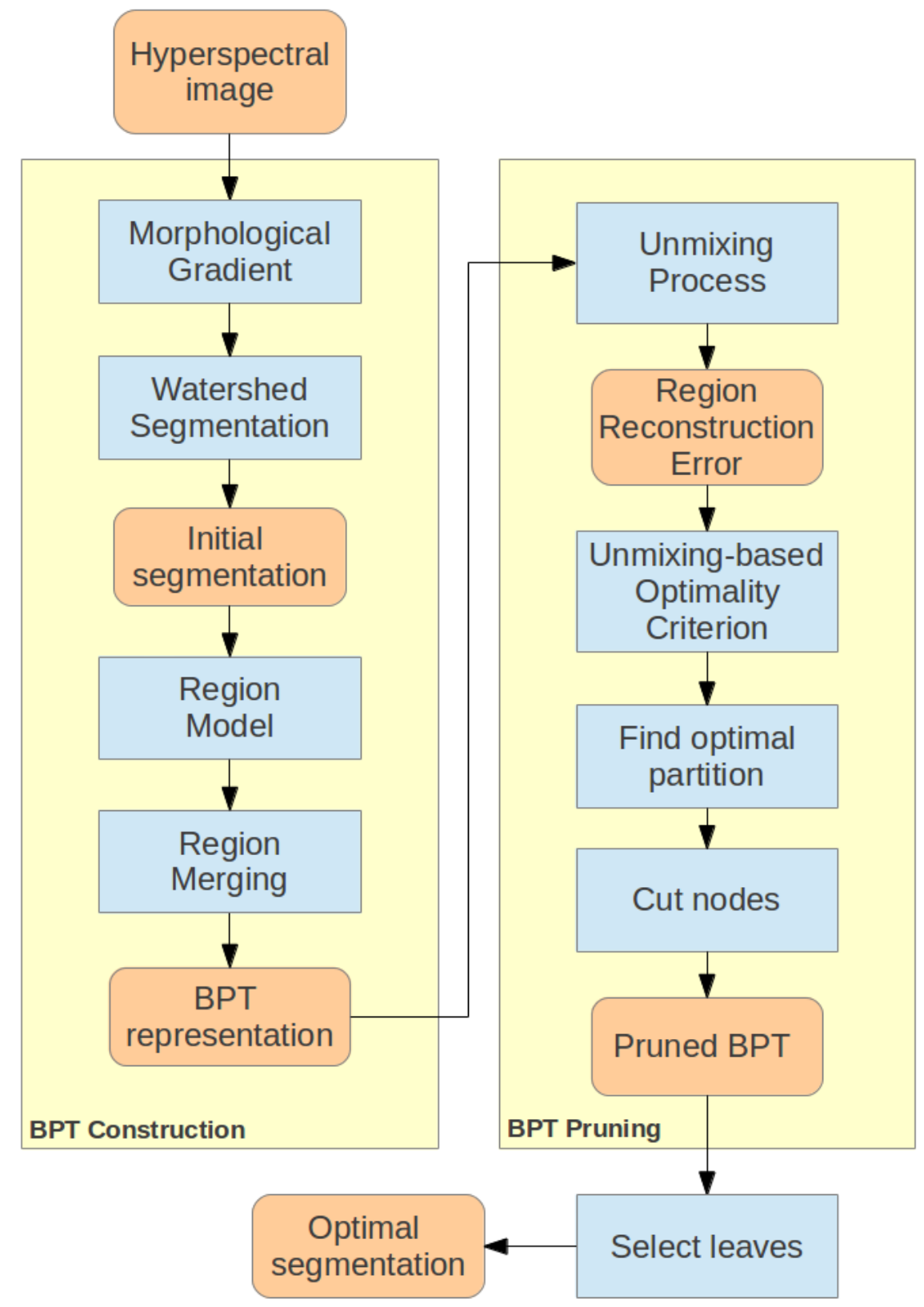

Fig. 3. Flowchart of the proposed novel methodology.

between two endmembers $\mathbf{e}_{k} \in \mathbf{E}_{\mathcal{R}_{\alpha}}$ and $\mathbf{e}_{l} \in \mathbf{E}_{\mathcal{R}_{\beta}}$. Once two regions merge into a new one, the set of endmembers for the new (larger) region is induced again by the given EIA. The rationale and originality of this spectral region model and merging criterion is to favour the grouping of neighbouring regions that are made of similar materials (endmembers). The proposed spectral merging criterion, as it is defined, strongly penalizes regions that do not contain the same materials, therefore it is fully adapted to the underlying motivation. 
2) Spectral-spatial region model and merging criterion: For each region $\mathcal{R}_{i}$ a set of $m_{i}$ endmembers $\mathbf{E}_{\mathcal{R}_{i}}=$ $\left[\mathbf{e}_{1}, \ldots, \mathbf{e}_{m_{i}}\right]$ is induced by some EIA, and their corresponding abundances, $\boldsymbol{\Phi}_{\mathcal{R}_{i}}=\left[\phi_{1}, \ldots, \phi_{m_{i}}\right]$, are estimated. Then, the spectral-spatial region model is defined as:

$$
\mathcal{M}_{\mathcal{R}_{i}} \stackrel{d}{=}\left\langle\mathbf{E}_{\mathcal{R}_{i}}, \bar{\phi}_{\mathcal{R}_{i}}\right\rangle
$$

where the tuple, $\left\langle\mathbf{E}_{\mathcal{R}_{i}}, \bar{\phi}_{\mathcal{R}_{i}}\right\rangle$, is composed by the set of endmembers, $\mathbf{E}_{\mathcal{R}_{i}}$ and their corresponding average fractional abundances, $\bar{\phi}_{\mathcal{R}_{i}}=\left[\bar{\phi}_{1}, \ldots, \bar{\phi}_{m_{i}}\right]$, such that $\bar{\phi}_{i}=\frac{1}{N_{\mathcal{R}_{i}}} \sum_{j=1}^{N_{\mathcal{R}_{i}}} \phi_{i}^{(j)}$, where $N_{\mathcal{R}_{i}}$ denotes the number of pixels in the region $\mathcal{R}_{i}$, and $\phi_{i}^{(j)}$ the fractional abundance of the $i$ th endmember for the $j$ th pixel. The spectral-spatial merging criterion between two regions $\mathcal{R}_{\alpha}$ and $\mathcal{R}_{\beta}$ modelled by (11) is given by the spectral-spatial dissimilarity between the set of endmembers and the corresponding average abundances of the two regions [41]:

$$
\begin{array}{r}
\mathcal{O}\left(\mathcal{M}_{\mathcal{R}_{\alpha}}, \mathcal{M}_{\mathcal{R}_{\beta}}\right) \stackrel{d}{=} s\left(\left\langle\mathbf{E}_{\mathcal{R}_{\alpha}}, \bar{\phi}_{\mathcal{R}_{\alpha}}\right\rangle,\left\langle\mathbf{E}_{\mathcal{R}_{\beta}}, \bar{\phi}_{\mathcal{R}_{\beta}}\right\rangle\right)= \\
\sum_{k, l} w_{k, l} d_{k, l},
\end{array}
$$

where $d_{k l}$ is the spectral angle distance between two endmembers, $\mathbf{e}_{k} \in \mathbf{E}_{\mathcal{R}_{\alpha}}$ and $\mathbf{e}_{l} \in \mathbf{E}_{\mathcal{R}_{\beta}}$, as it was defined above; and, $w_{k, l}$ is a weighting coefficient measuring the significance associated to $d_{k, l}$. The matrix of weighting coefficients, $W_{\alpha, \beta}=\left[w_{k l}\right], k=1, \ldots, m_{\alpha}, l=1, \ldots, m_{\beta}$, is calculated using the significance credit assignment algorithm (see Algorithm 1) introduced in [41] which is a version of the most similar highest priority principle [26], where the average fractional abundances, $\bar{\phi}_{\mathcal{R}_{\alpha}}$ and $\bar{\phi}_{\mathcal{R}_{\beta}}$ play the role of "significant credits" assigned to the spectral distances, $d_{k, l}$. The use of the proposed spectral-spatial merging criterion promotes the merging of regions containing similar materials and in similar proportions.

\section{B. Novel unmixing-based pruning criteria for segmentation}

We present now four new pruning criteria based on the spectral unmixing of the regions in the BPT representation of an hyperspectral image, in order to segment the image. The proposed unmixing-based pruning criteria are inspired in the Lagrangian formulation in (3), where the fitting function $D(\mathcal{R})$ is defined in terms of the unmixing reconstruction error (8). The two first proposed criteria follow the scheme in (3) but we are not imposing any constraint to the number of regions, so in strict terms the proposed criteria do not correspond to Lagrangian optimizations. However, the $\lambda$ term plays a similar role, weighting the trade-off between the unmixing reconstruction error and the partition complexity. The second two pruning strategies follow an analogous approach, but the optimization problem is formulated in terms of the lattice supremum operator, $\bigvee$, instead of the arithmetic sum operator, $\sum$. Together with the four unmixing-based pruning criteria, we provide the algorithms to solve the optimization problems.

1) Arithmetic definition of the unmixing-based pruning strategies: Let $\mathcal{P}$ be a partition of the image (a pruning of the BPT) and $\Omega$ be the set of all possible partitions. Then, we propose a first unmixing-based pruning criterion defined as the partition minimizing the overall average RMSE regularized by the number of regions in the partition:

$$
\mathcal{P}_{\sum}^{\star} \text { avg }=\arg \min _{\mathcal{P} \in \Omega} \frac{1}{N} \sum_{\mathcal{R} \in \mathcal{P}} \sum_{\mathbf{r} \in \mathcal{R}} \epsilon_{\mathcal{R}}(\mathbf{r}, \hat{\mathbf{r}})+\lambda|\mathcal{P}|,
$$




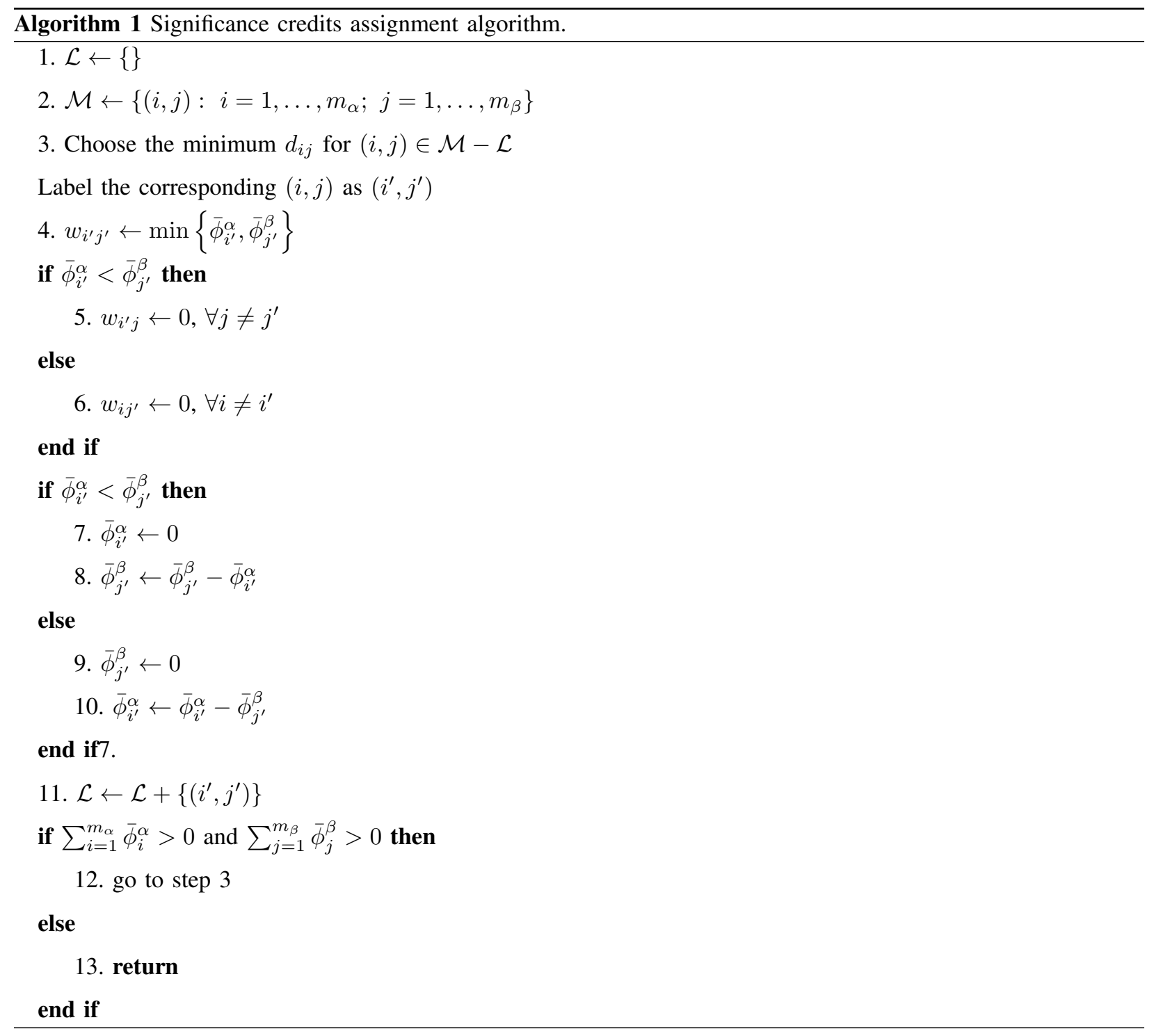

where $N$ denotes the number of pixels in the image, $|\mathcal{P}|$ denotes the number of regions in the partition and $\epsilon_{\mathcal{R}}(\mathbf{r}, \hat{\mathbf{r}})$ is the RMSE (8) for the pixel $\mathbf{r}$ with respect to the reconstructed pixel $\hat{\mathbf{r}}=\sum_{i=1}^{m} \hat{\mathbf{e}}_{\mathbf{i}} \hat{\phi}_{i}$ calculated using the set of endmembers $\hat{\mathbf{E}}_{\mathcal{R}}$ and the fractional abundances $\hat{\mathbf{\Phi}}_{\mathcal{R}}$ obtained from the unmixing of the region $\mathcal{R}$. This expression can be obtained from (3) by setting the fitting and complexity functions to be $D(\mathcal{R})=1 / N \sum_{\mathbf{r} \in \mathcal{R}} \epsilon_{\mathcal{R}}(\mathbf{r}, \hat{\mathbf{r}})$ and $C(\mathcal{R})=1$.

Similarly, we define a second criterion as the partition minimizing the weighted average of the maximum RMSE of the regions in the partition, regularized by the number of regions in the partition:

$$
\mathcal{P}_{\sum \max }^{\star}=\arg \min _{\mathcal{P} \in \Omega} \frac{1}{N} \sum_{\mathcal{R} \in \mathcal{P}} N_{\mathcal{R}} \max \epsilon_{\mathcal{R}}(\mathbf{r}, \hat{\mathbf{r}})+\lambda|\mathcal{P}|,
$$

where $N_{\mathcal{R}}$ denotes the number of pixels in the region $\mathcal{R}$. In this case, the expression can be obtained by setting $D(\mathcal{R})=N_{\mathcal{R}} /|\mathcal{P}| \max \epsilon_{\mathcal{R}}(\mathbf{r}, \hat{\mathbf{r}})$ and $C(\mathcal{R})=1$. 
Given the BPT representation, $\mathcal{T}(\mathcal{I})$, of the hyperspectral image $\mathcal{I}$ and the value of the regularization parameter, $\lambda$, these two optimization problems (13) and (14) can be solved by dynamic programming as it was proved in [20]. Algorithm 2 works in a recursive way starting from the root of the BPT hierarchy. The energy of the root node is calculated using the reconstruction error function, $D(\mathcal{R})$, and the complexity function, $C(\mathcal{R})$. Then, it makes a recursive call to obtain the optimal cut of the root children. Once the optimal cuts are obtained, that is, the energy and the optimal partition of each of the children; the energy of the root is compared to the addition of the energies of the children. If the energy of the root is lower than the sum of the children's energies, the optimal partition is given by the root node. Otherwise, the optimal partition is given by the union of the optimal cuts of the children. The optimalCut function works in a similar way, traversing the tree top-down by successive recursive calls up to find the leaves. Then, the recursive function returns the calls in a bottom-up till reaching the root node.

2) Lattice definition of the unmixing-based pruning strategies: We also propose two novel unmixing-based pruning criteria in base to the following optimization problem:

$$
\mathcal{P}^{\star}=\arg \min _{\mathcal{P} \in \Omega} \bigvee_{\mathcal{R} \in \mathcal{P}}[D(\mathcal{R})+\lambda C(\mathcal{R})]
$$

where the $\sum$ symbol in the conventional optimization formulation (3) has been replaced by the lattice operator $\bigvee$. Eq. (15) characterizes a partition by bounds on the fitting and complexity functions. The optimal partition is the one that minimizes the regularized combination of both bounds.

Using this lattice formulation, we define a third unmixing-based pruning criterion as:

$$
\mathcal{P}_{\bigvee}^{\star} \max =\arg \min _{\mathcal{P} \in \Omega} \bigvee_{\mathcal{R} \in \mathcal{P}}\left[\max \epsilon_{\mathcal{R}}(\mathbf{r}, \hat{\mathbf{r}})+\frac{\lambda}{N_{\mathcal{R}}}\right],
$$

with $D(\mathcal{R})=\max \epsilon_{\mathcal{R}}(\mathbf{r}, \hat{\mathbf{r}})$, and $C(\mathcal{R})=1 / N_{\mathcal{R}}$. The pruning criterion in (16) finds a partition that minimizes the upper bound on the partition's reconstruction error and at the same time maximizes the lower bound on the size of the regions in the partition, where the trade-off is governed by the $\lambda$ parameter.

In a similar fashion, we propose the following fourth unmixing-based pruning criterion:

$$
\mathcal{P}_{\bigvee}^{\star} \text { avg }=\arg \min _{\mathcal{P} \in \Omega} \bigvee_{\mathcal{R} \in \mathcal{P}}\left[\frac{1}{N_{\mathcal{R}}} \sum_{\mathbf{r} \in \mathcal{R}} \epsilon_{\mathcal{R}}(\mathbf{r}, \hat{\mathbf{r}})+\frac{\lambda}{N_{\mathcal{R}}}\right],
$$

with $D(\mathcal{R})=\frac{1}{N_{\mathcal{R}}} \sum_{\mathbf{r} \in \mathcal{R}} \epsilon_{\mathcal{R}}(\mathbf{r}, \hat{\mathbf{r}})$, and $C(\mathcal{R})=1 / N_{\mathcal{R}}$. The pruning criterion in (16) finds a partition that minimizes the upper bound on the average reconstruction error of the regions and at the same time maximizes the lower bound on the size of the regions in the partition, where the trade-off is governed by the $\lambda$ parameter.

The algorithm to solve (16) and (17) is analogous to Algorithm 2. The pseudocode of the algorithm is depicted in Algorithm 3. Both, the algorithm and the proof that it is able to find the optimal partition are analogous to the Algorithm 2 and the proof provided in [20], where the lattice operators $\bigvee$ and max replace the arithmetic operators $\sum$ and + .

3) Use of a size constraint: It is sometimes interesting to constrain the set of valid partitions, $\Omega$, to those containing regions above a minimum spatial size. For instance, the segmentation of the image could be later used for applications that require a minimum number of pixels to work, i.e. estimates of statistical parameters. In these 


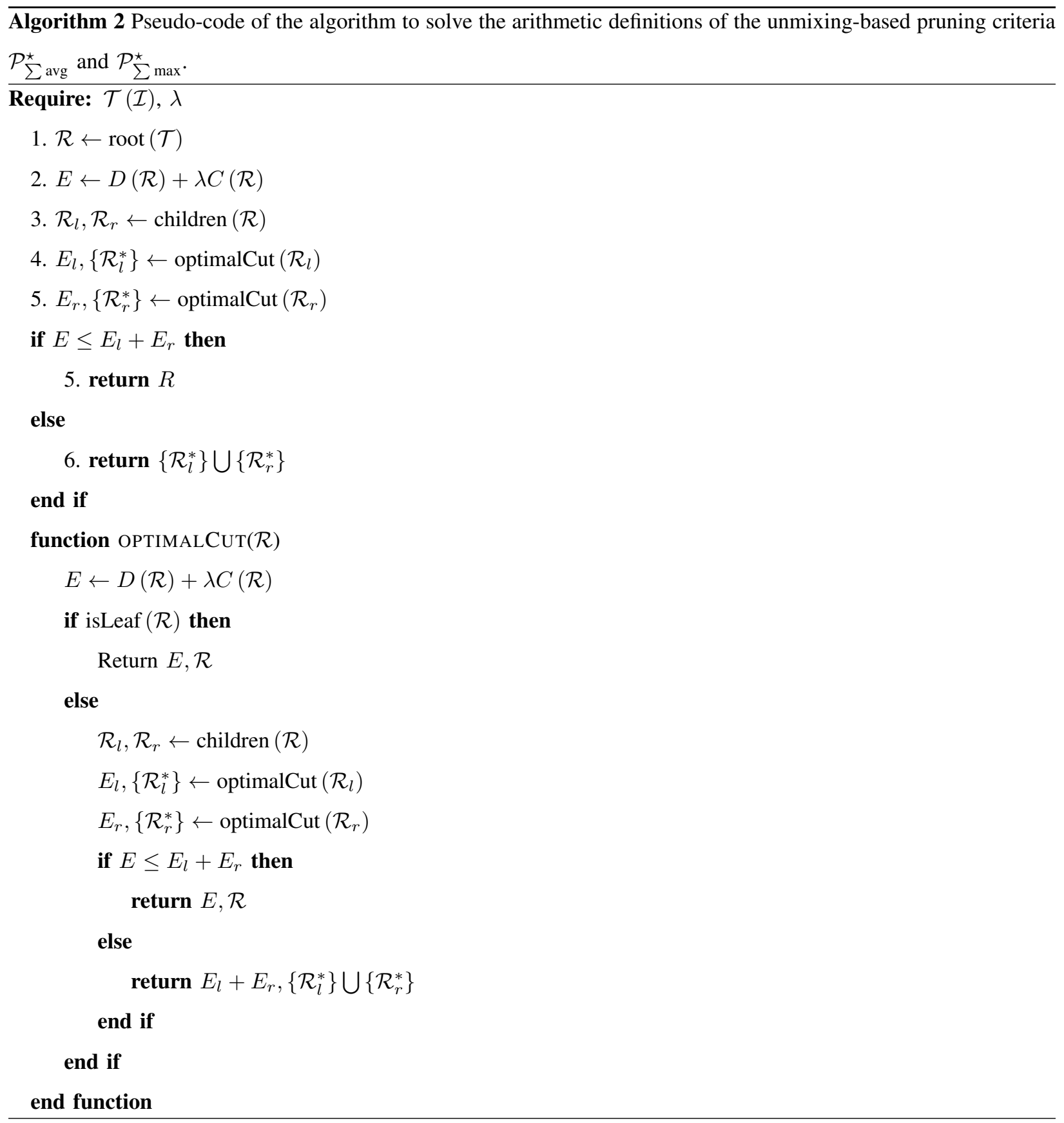

cases, the set $\Omega$ of valid partitions in the formulation of the optimization problems is replaced by the subset of size-constrained valid partitions, $\Omega_{c}$ :

$$
\Omega_{c}=\left\{\mathcal{P} \in \Omega \text {, s.t. } \forall \mathcal{R} \in \mathcal{P}, N_{\mathcal{R}} \geq c\right\},
$$

where $N_{\mathcal{R}}$ denotes the number of pixels in region $\mathcal{R}$ and $c \geq 0$ is a threshold on the region size. If $c=0$, the term (18) has no effect and the pruning criterion is considered to be unconstrained. 


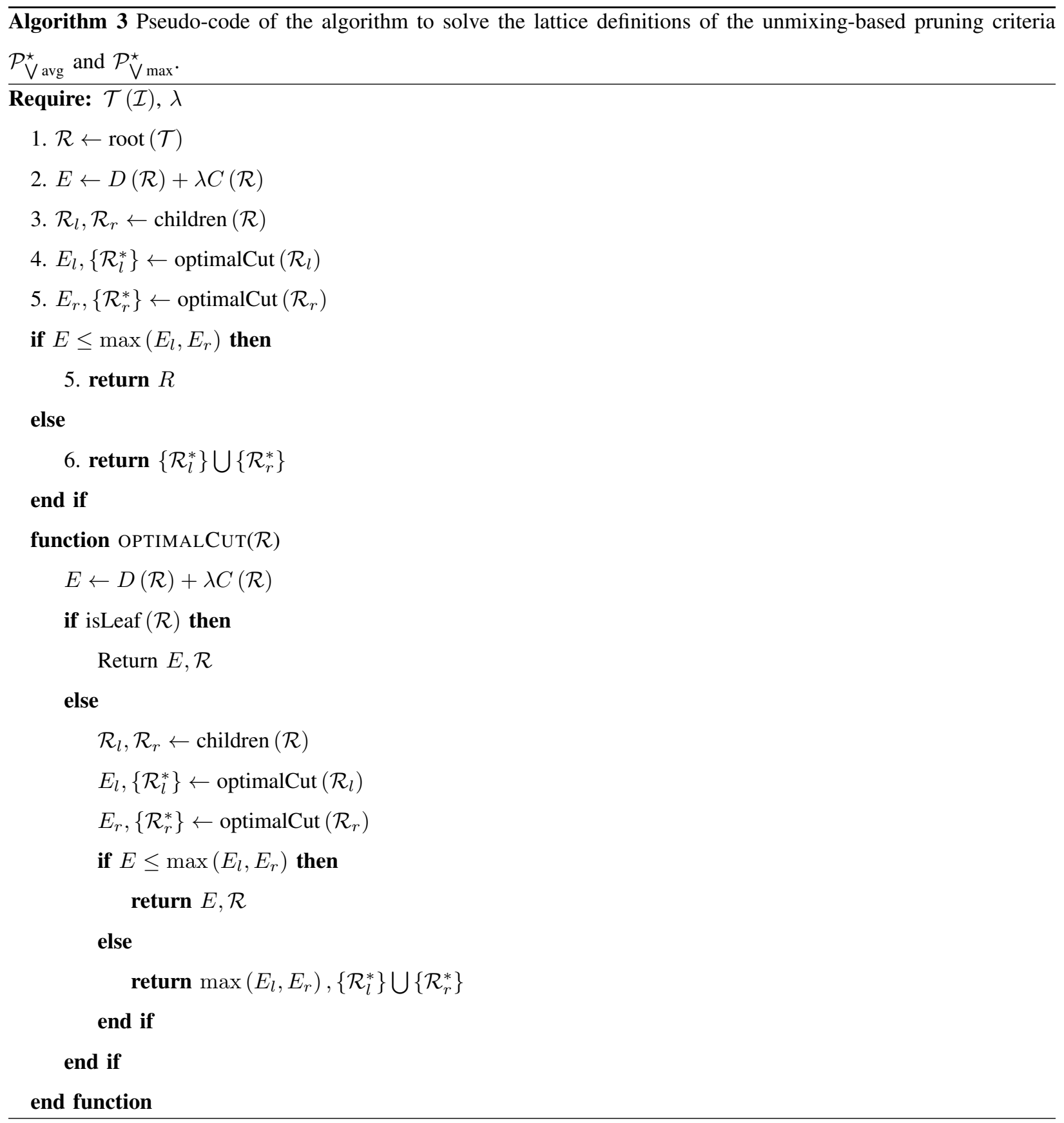

\section{Proposed methodology}

Fig. 3 shows the flow diagram of the proposed general methodology to obtain an optimal segmentation from a hyperspectral image, by pruning the BPT representation of the image using the information provided by the spectral unmixing process. The procedure is as follows:

Step one. An initial oversegmentation of the image is provided, instead of starting from the entire set of pixels, to speed-up the BPT building. The initial segmentation should contain regions small enough not to encompass 
"actual" regions, and accurate enough to be able to reconstruct those regions with a good accuracy. Hence, starting from an undersegmented image should be avoided at all cost as regions will not be allowed to split later on. On this purpose, we propose to obtain the initial partition by a Watershed segmentation of the original image [32]. First, we calculate the supremum of the component-wise morphological gradient from the original image, and then we apply a classical Watershed onto this gradient map. Finally, we set all the resulting border pixels to their respective most similar connected regions as it is explained in [34].

Step two. A BPT representation of the image is obtained using a given region model and a region merging criterion compatible with it. We propose to use either, the spectral region model (9) and merging criterion (10) or the spectral-spatial region model (11) and merging criterion (12) given in Section IV-A. In order to do that, a spectral unmixing process is run independently for each region (see Fig. 4). First, the virtual dimensionality $\delta$ of the region $\mathcal{R}$ is computed using the Hyperspectral Signal Subspace Estimation (Hysime) algorithm [6]. The value of $\delta$ works as an estimation of the number $m$ of endmembers present in the region. If the region is too small to correctly estimate the number of endmembers (due to the presence of close to singular covariance matrices during the application of the Hysime algorithm), that is, if $\delta=0$ or $\delta>N_{\mathcal{R}}$, being $N_{\mathcal{R}}$ the number of pixels in the region, then its region model $\mathcal{M}_{\mathcal{R}}$ is set to the mean spectrum of the region as in (1). This happens in very small and homogeneous regions, so the mean spectrum $\overline{\mathbf{r}}$ acts as a single endmember. Otherwise, an EIA is run over the $N_{\mathcal{R}}$ pixels of the region to induce the corresponding set of endmembers. To overcome the stochastic part of most of the EIAs, the induction algorithm is run a number of times $k$ for each region, and the set of endmembers yielding the larger simplex volume [43], $V_{k}(\mathbf{E})$, among the $k$ trials is retained. If the spectral region model is selected to build the BPT representation, the region model is defined by these endmembers as it is described in (9). If the spectral-spatial region model is selected, the FCLSU is conducted and the fractional abundances of the induced endmembers are estimated for each pixel in the region. The region model is then defined by the endmembers and their average fractional abundances as it is described in (11). The population of the tree with the unmixing information could be computationally expensive, but once the BPT has been populated it can be stored and any posterior processing of the BPT representation is usually very fast. This trade-off is common in the analysis of images by means of tree representations.

Step three. The reconstruction error of each region is calculated by the RMSE (8) given the set of endmembers and corresponding abundances obtained from the pixels of each region. Note that if any of the two proposed unmixingbased region models are used to build the BPT representation, this information is already calculated in step 2. If not, for instance, when using the first-order parametric region model (1), the spectral unmixing process defined above should be run for each region in order to induce the endmembers and estimate the fractional abundances.

Step four. Finally, one of the four proposed unmixing-based pruning criteria (see Sec. IV-B) is applied to prune the BPT and find the nodes defining the optimal segmentation according to the spectral unmixing reconstruction error and the regularization term. 


\section{MATERIALS AND EXPERIMENTAL METHODOLOGY}

\section{A. Hyperspectral datasets}

Two real hyperspectral data sets have been used in our experiments. Their selection is supported by the fact that these scenes have been widely used to validate hyperspectral segmentation and spectral unmixing applications, and currently constitute benchmarks used to validate new algorithms thanks to the availability of reliable reference information. The considered scenes can be summarized as follows:

The Pavia University hyperspectral image was collected by the ROSIS-03 sensor over the facilities of the University of Pavia in Italy. After discarding pixels with no information and noisy spectral bands, the image has a spatial size of $610 \times 340$ pixels with a spatial resolution of $1.3 \mathrm{~m}$ per pixel, and 93 spectral bands comprised in the range of 430-860 nm. Fig. 5(a) shows a false color representation of the Pavia University scene. The scene shows an urban area comprised of different buildings, parking lots, roads and other typical human-made constructions, together with trees, green areas and bare soil.

The Cuprite hyperspectral scene [18] was acquired by the NASA's AVIRIS sensor and covers the Cuprite mining district in western Nevada, USA. This sensor collects data in 224 contiguous spectral bands with a bandwidth of $0.10 \mu \mathrm{m}$ in the range of $0.4-2.5 \mu \mathrm{m}$. After removing noisy bands due to atmospheric water absorption 200 bands remain. Each pixel represents a $20 \mathrm{~m}^{2}$ square cell. The data used in the experiments is a $250 \times 190$ subset of the original scene covering the mineralogical region of interest. Fig. 5(b) shows a false color representation of the Cuprite scene and the subset used for experiments. The scene is well-known and widely used in hyperspectral community thanks to the extensive reference information available for this scene from the United States Geological Survey (USGS) ${ }^{1}$.

\section{B. Experimental methodology}

This section describes the procedure adopted to conduct the analysis of the two aforementioned hyperspectral scenes. Specifically, we describe the steps followed in order to build and prune the BPT in our experiments, and the quantitative measures employed to compare the different segmentation-oriented pruning criteria.

For each dataset, we built three independent BPT representations corresponding to each of the three presented region models and corresponding merging criteria: a) the first-order parametric region model (1) and the spectral angle distance-based merging criterion (2), b) the spectral region model (9) and the spectral distance-based merging criterion (10), and c) the spectral-spatial region model (11) and the spectral-spatial distance-based merging criterion (12). In all cases, we set the priority term [9], [36] to 0.15. For each of the three BPT representations, we populated the tree with the endmembers and the fractional abundances obtained from an unmixing process run in each node of the BPT representations, as it was explained in Section IV-C. In order to do that, we employed the Vertex Component Analysis (VCA) algorithm [27] to induce the endmembers. In the case of the BPT representations using the spectral and spectral-spatial region models, the unmixing information is obtained during the BPT construction.

\footnotetext{
${ }^{1}$ http://speclab.cr.usgs.gov/cuprite.html
} 
TABLE I

COMPUTATIONAL TIMES OF THE CONSTRUCTION AND UNMIXING POPULATION OF THE BPT REPRESENTATION OF PAVIA UNIVERSITY AND CUPRITE SCENES, USING THE THREE DIFFERENT REGION MODELS AND MERGING STRATEGIES.

\begin{tabular}{|c|c|c|c|}
\cline { 2 - 4 } \multicolumn{1}{c|}{} & First-order parametric & Spectral & Spectral-spatial \\
\hline Pavia University & $98^{\prime} 48^{\prime \prime}$ & $98^{\prime} 16 ”$ & $104^{\prime} 22^{\prime \prime}$ \\
\hline Cuprite & $27^{\prime} 58^{\prime \prime}$ & $28^{\prime} 06^{\prime \prime}$ & $30^{\prime} 33^{\prime \prime}$ \\
\hline
\end{tabular}

In the case of the BPT representation using the first-order parametric region model, the population is done after the construction of the BPT representation. The computational times required to build the BPT representations and populate them with the unmixing information using a 64bits 4 core Intel $\mathrm{Xeon}(\mathrm{R}) \mathrm{CPU}$ at $2.80 \mathrm{GHz}$ are presented in Table I. The computational time linearly scales with the size of the image, and is mainly due to the unmixing population, since the computational cost of the BPT construction and the posterior pruning is almost negligible. We plan to develop computationally efficient implementations (using high performance computing architectures) of the proposed strategies for the construction and unmixing population of the tree representation in order to reduce the computational burden.

Then, for each of the BPT representations, we independently pruned them by means of the four proposed pruning approaches based on the arithmetic optimization formulation (13) and (14), named as "Proposed SUM(AVG)" and "Proposed SUM(MAX)", respectively; and on the lattice optimization formulation (16) and (17), named as "Proposed SUP(MAX)" and "Proposed SUP(AVG)", respectively. We compared the results of the proposed pruning strategies to the two common strategies presented in section II-B, the height and region pruning strategies. We also compared to the pruning criterion proposed by Valero in [37], named as "Valero SUM(SID)". In order to make a fair comparison, we fixed a set of expected number of regions and, in the case of the "Valero SUM(SID)" and the proposed pruning criteria, we experimentally obtained the $\lambda$ values that yield to partitions with a number of regions close to the expected ones. In the case of the height-based pruning criterion we obtained the height that yield to partitions that better approximated the desired number of regions. We run eight independent experiments for each pruning criteria, BPT representation and dataset, where the expected number of regions in the optimal partition, $\left|\mathcal{P}^{*}\right|$, was respectively set to $\left|\mathcal{P}^{*}\right|=[5,10,20,35,50,75,150,500]$ for the Cuprite scene, and $\left|\mathcal{P}^{*}\right|=[5,10,40,75,100,225,350,850]$ for the Pavia University scene. The selected values were arbitrarily chosen.

In order to quantitatively compare the segmentations obtained by the different pruning criteria, we compared the original image, $\mathcal{I}$, to the one obtained by the unmixing reconstruction, $\hat{\mathcal{I}}=\hat{\mathbf{E}} \hat{\boldsymbol{\Phi}}$, calculated from the partitions obtained by the different BPT representation models, pruning criteria and expected partition sizes. The reconstruction was made piece-wise, where the endmembers and fractional abundances obtained in each region of a given segmentation were used to reconstruct the pixels of that region. We made use of four different image reconstruction quality measures. Two of them, the average RMSE and the average SAD, measure the average Euclidean and 
angular spectral error, respectively:

$$
\operatorname{avgRMSE}(\mathcal{I}, \hat{\mathcal{I}})=\frac{1}{N} \sum_{i=1}^{N} \epsilon\left(\mathbf{r}_{i}, \hat{\mathbf{r}}_{i}\right),
$$

where $\epsilon(\mathbf{r}, \hat{\mathbf{r}})$ is the RMSE (8) between a pixel in the original image, $\mathbf{r} \in \mathcal{I}$, and the corresponding one in the reconstructed image, $\hat{\mathbf{r}} \in \hat{\mathcal{I}}$.

$$
\operatorname{avgSAD}(\mathcal{I}, \hat{\mathcal{I}})=\frac{1}{N} \sum_{i=1}^{N} \operatorname{SAD}\left(\mathbf{r}_{i}, \hat{\mathbf{r}}_{i}\right),
$$

where $\operatorname{SAD}\left(\mathbf{r}_{i}, \hat{\mathbf{r}}_{i}\right)$ is the spectral angle distance (2). The third one, the average $\mathrm{Q}$, measures the average correlation between the original and the reconstructed images:

$$
\operatorname{avgQ}(\mathcal{I}, \hat{\mathcal{I}})=\frac{\sigma_{\mathcal{I} \hat{\mathcal{I}}}}{\sigma_{\mathcal{I}} \sigma_{\hat{\mathcal{I}}}} \frac{2 \mu_{\mathcal{I}} \mu_{\hat{\mathcal{I}}}}{\mu_{\mathcal{I}}^{2}+\mu_{\hat{\mathcal{I}}}^{2}} \frac{2 \sigma_{\mathcal{I}} \sigma_{\hat{\mathcal{I}}}}{\sigma_{\mathcal{I}}^{2}+\sigma_{\hat{\mathcal{I}}}^{2}}
$$

where $\mu_{\mathcal{I}}$ and $\mu_{\hat{\mathcal{I}}}$ are the mean $q$-dimensional vectors of the original and reconstructed images respectively; $\sigma_{\mathcal{I}}$ and $\sigma_{\hat{\mathcal{I}}}$ denote the variances, and $\sigma_{\mathcal{I} \hat{\mathcal{I}}}$ the covariance. The last one is the ERGAS quality measure which evaluates both spectral and spatial divergences:

$$
\operatorname{ERGAS}(\mathcal{I}, \hat{\mathcal{I}})=100 \sqrt{\frac{1}{N} \sum_{i=1}^{N}\left(\frac{\epsilon\left(\mathbf{r}_{i}, \hat{\mathbf{r}}_{i}\right)}{\mu_{\mathbf{r}_{i}}}\right)^{2}},
$$

where $\mu_{\mathbf{r}_{i}}$ denotes the mean value of the pixel $\mathbf{r}_{i}$.

\section{Results using Pavia University scene}

\section{A. Reconstruction errors}

Figs. 6-8 show the quantitative reconstruction quality measures of the different pruning criteria applied over the BPT representations obtained by the first-order parametric model, the spectral model and the spectral-spatial model representations of the Pavia University scene, respectively. Each point in the plots represents a partition obtained by each of the pruning strategies over the corresponding BPT. In order to compare them, we plot the quality measure with respect to the number of regions contained in each partition. The conventional region-based pruning strategy is not shown since the quality measures obtained for it are very poor in all cases, making it difficult to visualize the differences among the other methods if we include it in the plots.

The four proposed unmixing-based merging criteria outperform the other methods in all the cases. The differences among the four proposed pruning criteria are small, being the "Proposed SUM(AVG)" (13) consistently the best. Nevertheless, the two proposed lattice-based pruning criteria present similar quality values compared to the arithmetic-based ones, while they promote low upper bounds to the spectral RMSE reconstruction error, and supposedly to the other aforementioned spectral reconstruction errors as well. With regards to the different region models, the ones using the unmixing information to build the BPT representation present better quantitative results than the first-order parametric model. This is especially relevant for the conventional and the "Valero SUM(SID)" pruning criteria that make no use of the unmixing information, showing that the use of the spectral unmixing information either to obtain the BPT representation and/or to prune the BPT is absolutely meaningful. 


\section{B. Segmentation results}

Fig.VI-B shows the segmentations obtained by the four proposed unmixing-based pruning criteria and the one by Valero, for the spectral-spatial BPT representation of the Pavia University scene. The top row shows segmentations with about 50 regions, while the bottom row shows segmentations with approximately 100 regions. It can be appreciated that the regions comprise structures which are spatially meaningful, for instance, grouping the university (big blue building), the parking lots and other visible structures. However, there are not many visual differences among the five approaches. The only noticeable difference is that the "Proposed SUM(AVG)" and the "Proposed MAX(AVG)" approaches do not change much when the spatial regularization term is favoured, compared to the other three approaches, which produce segmentations with smaller regions. This can be better understood looking at the reconstruction quality measures (8), where these two pruning criteria stabilize around segmentations with approximately 50 regions. It means that the $\lambda$ parameter should be severely increased to obtain more oversegmented partitions.

\section{RESUlts USING CUPRITE SCENE}

\section{A. Reconstruction errors}

Figs. 10-12 show the quantitative reconstruction quality measures of the different pruning criteria applied over the BPT representations obtained by the first-order parametric model, the spectral model and the spectral-spatial model representations of the Cuprite scene, respectively. The obtained quantitative results present a similar trend to the obtained for the Pavia University scene. The main difference is that the "Proposed SUP(AVG)" (17) pruning criterion is doing worse than the other proposed pruning criteria. This can be explained by a limitation in the minimization of the upper bound on the average region error that could be achieved for this dataset. However, the other three proposed approaches consistently outperform the one by Valero and the conventional ones, being the "Proposed SUM(AVG)" the one showing the best results again.

\section{B. Segmentation results}

Fig.VII-B shows the segmentations obtained by the four proposed unmixing-based pruning criteria and the one by Valero, for the spectral-spatial BPT representation of the Cuprite scene. As with Pavia University scene, the top row shows segmentations with about 50 regions, while the bottom row shows segmentations with approximately 100 regions. Being a scene of a natural landscape, it is difficult to appreciate if the regions are spatially meaningful or not. Visually, it can be appreciate that the "Proposed SUM(AVG)" and the "Valero SUM(SID)" approaches get similar segmentations. The same observation happens for the "Proposed SUM(MAX)" and the "Proposed SUP(MAX)". However, the "Proposed SUP(AVG)" is the one achieving the most different segmentation. The segmentation obtained by this approach contains one large region that comprises approximately two thirds of the image, and the remaining area is divided in small regions. This highly oversegmented area can be identified as the mining district, where most of the spectral variability due to mineral takes place. 


\section{CONCLUSIONS AND FUTURE RESEARCH LINES}

In this paper, we have developed a new strategy for the representation of hyperspectral images using binary partition trees and concepts from spectral unmixing. The presented approach addresses hyperspectral image segmentation and unmixing in a synergistic fashion, i.e., by combining the properties of the two aforementioned methods into a unified framework for the first time in the literature. The presented strategy has been evaluated using reference hyperspectral scenes representing two contexts, urban areas and natural landscapes, at different spatial and spectral resolutions. Overall, the segmentations obtained for the proposed approaches are meaningful and valuable for hyperspectral image analysis. The four proposed unmixing-based pruning criteria yield to segmentations that outperformed the approach by Valero and the conventional height and region-based pruning criteria in terms of reconstruction quality. The "Proposed SUM(AVG)" (13) pruning criteria consistently outperformed the other ones. In general, the use of information coming from the unmixing process either in the construction of the BPT representation, by means of the spectral and spectral-spatial region models and merging criteria, or in the pruning of the BPT, by means of the four proposed unmixing-based pruning criteria, has a clear positive impact in the quality of the obtained segmentations.

Although the proposed method has been shown to be a relevant new framework for hyperspectral data interpretation, there are some aspects that may present challenges over time and which deserve a more extensive evaluation. Among them, we list the possibility to use other unmixing-based fitting functions in the definition of the pruning criterion or the evaluation using additional hyperspectral scenes. The proposed approach was found to be useful not only to perform segmentation by taking into account the sub-pixel nature of mixed pixels, but also to perform spectral unmixing using a local-to-global approach in which the optimization criteria is based on the minimization of reconstruction errors at a local scale, which results in an overall minimization of reconstruction errors that is highly appealing for spectral unmixing applications. This avenue will be further investigated. We also plan to develop computationally efficient implementations (using high performance computing architectures) of the proposed strategies for the construction and unmixing population of the tree representation in order to reduce its computational burden.

\section{ACKNOWLEDGEMENTS}

We would like to thank Prof. Salembier and Dr. Valero for pointing us to the pruning criteria introduced in the Ph.D. Dissertion of Dr. Valero. The Pavia University dataset has been provided by Prof. Gamba from the Telecommunications and Remote Sensing Laboratory, Pavia University (Italy). We gratefully thank the Associate Editor who handled our manuscript and the Anonymous Reviewers for their outstanding comments and suggestions.

\section{REFERENCES}

[1] T. Akgun, Y. Altunbasak, and R.M. Mersereau. Super-resolution reconstruction of hyperspectral images. IEEE Transactions on Image Processing, 14(11):1860-1875, 2005.

[2] N. Alajlan, Y. Bazi, F. Melgani, and R.R. Yager. Fusion of supervised and unsupervised learning for improved classification of hyperspectral images. Information Sciences, 217(0):39 - 55, 2012. 
[3] J.A. Benediktsson, J. Chanussot, and W.M. Moon. Very high-resolution remote sensing: Challenges and opportunities [point of view]. Proceedings of the IEEE, 100(6):1907-1910, 2012.

[4] K. Bernard, Y. Tarabalka, J. Angulo, J. Chanussot, and J.A. Benediktsson. Spectral spatial classification of hyperspectral data based on a stochastic minimum spanning forest approach. IEEE Transactions on Image Processing, 21(4):2008-2021, 2012.

[5] G. Bilgin, S. Erturk, and T. Yildirim. Segmentation of hyperspectral images via subtractive clustering and cluster validation using one-class support vector machines. IEEE Transactions on Geoscience and Remote Sensing, 49(8):2936-2944, 2011.

[6] J.M. Bioucas-Dias and J.M.P. Nascimento. Hyperspectral subspace identification. IEEE Transactions on Geoscience and Remote Sensing, 46(8):2435-2445, 2008.

[7] J.M. Bioucas-Dias, A. Plaza, N. Dobigeon, M. Parente, Qian Du, P. Gader, and J. Chanussot. Hyperspectral unmixing overview: Geometrical, statistical, and sparse regression-based approaches. IEEE Journal of Selected Topics in Applied Earth Observations and Remote Sensing, 5(2):354-379, 2012.

[8] F. Bovolo, L. Bruzzone, and L. Carlin. A novel technique for subpixel image classification based on support vector machine. IEEE Transactions on Image Processing, 19(11):2983-2999, 2010.

[9] F. Calderero and F. Marques. Region merging techniques using information theory statistical measures. IEEE Transactions on Image Processing, 19(6):1567-1586, June 2010.

[10] G. Cao, L.R. Bachega, and C.A. Bouman. The sparse matrix transform for covariance estimation and analysis of high dimensional signals. IEEE Transactions on Image Processing, 20(3):625-640, 2011.

[11] I. Dopido, A. Villa, A. Plaza, and P. Gamba. A quantitative and comparative assessment of unmixing-based feature extraction techniques for hyperspectral image classification. IEEE Journal of Selected Topics in Applied Earth Observations and Remote Sensing, 5(2):421-435, 2012.

[12] A. Erturk and S. Erturk. Unsupervised segmentation of hyperspectral images using modified phase correlation. IEEE Geoscience and Remote Sensing Letters, 3(4):527-531, 2006.

[13] A.F.H. Goetz. Three decades of hyperspectral remote sensing of the earth: A personal view. Remote Sensing of Environment, 113, Supplement 1(0):S5 - S16, 2009. Imaging Spectroscopy Special Issue.

[14] A.F.H. Goetz, G. Vane, J.E. Solomon, and B.N. Rock. Imaging spectrometry for earth remote sensing. Science, 228(4704):1147-1153, 1985.

[15] M. Golbabaee, S. Arberet, and P. Vandergheynst. Compressive source separation: Theory and methods for hyperspectral imaging. IEEE Transactions on Image Processing, 22(12):5096-5110, 2013.

[16] M. Graña and M.A. Veganzones. An endmember-based distance for content based hyperspectral image retrieval. Pattern Recognition, 45(9):3472-3489, 2012.

[17] M. Graña, I. Villaverde, J.O. Maldonado, and C. Hernandez. Two lattice computing approaches for the unsupervised segmentation of hyperspectral images. Neurocomputing, 72(10-12):2111 - 2120, 2009.

[18] R.O. Green, M.L. Eastwood, C.M. Sarture, T.G. Chrien, M. Aronsson, B.J. Chippendale, J.A. Faust, B.E. Pavri, C.J. Chovit, M. Solis, M.R. Olah, and O. Williams. Imaging spectroscopy and the airborne visible/infrared imaging spectrometer (aviris). Remote Sensing of Environment, 65(3):227-248, 1998.

[19] J.B. Greer. Sparse demixing of hyperspectral images. IEEE Transactions on Image Processing, 21(1):219-228, 2012.

[20] L. Guigues, J.P. Cocquerez, and H. Men. Scale-sets image analysis. International Journal of Computer Vision, 68(3):289-317, 2006.

[21] B. Guo, S.R. Gunn, R.I. Damper, and J.D.B. Nelson. Customizing kernel functions for SVM-Based hyperspectral image classification. IEEE Transactions on Image Processing, 17(4):622-629, 2008.

[22] D.C. Heinz and C.-I. Chang. Fully constrained least squares linear spectral mixture analysis method for material quantification in hyperspectral imagery. IEEE Transactions on Geoscience and Remote Sensing, 39(3):529-545, 2001.

[23] A. Ifarraguerri and C.-I. Chang. Unsupervised hyperspectral image analysis with projection pursuit. IEEE Transactions on Geoscience and Remote Sensing, 38(6):2529-2538, 2000.

[24] N. Keshava and J.F. Mustard. Spectral unmixing. Signal Processing Magazine, IEEE, 19(1):44-57, 2002.

[25] C.L. Lawson. Solving Least Squares Problems. Prentice Hall, 1974.

[26] J. Li, J.Z. Wang, and G. Wiederhold. Irm: Integrated region matching for image retrieval. In Proceedings of the Eighth ACM International Conference on Multimedia, MULTIMEDIA '00, pages 147-156, New York, NY, USA, 2000. ACM. 
[27] J.M.P. Nascimento and J.M.B. Dias. Vertex component analysis: a fast algorithm to unmix hyperspectral data. IEEE Transactions on Geoscience and Remote Sensing, 43(4):898-910, 2005.

[28] S. Piqueras, L. Duponchel, R. Tauler, and A. de Juan. Resolution and segmentation of hyperspectral biomedical images by multivariate curve resolution-alternating least squares. Analytica Chimica Acta, 705(1-2):182 - 192, 2011.

[29] A. Plaza, J.A. Benediktsson, J.W. Boardman, J. Brazile, L. Bruzzone, G. Camps-Valls, J. Chanussot, M. Fauvel, P. Gamba, A. Gualtieri, M. Marconcini, J.C. Tilton, and G. Trianni. Recent advances in techniques for hyperspectral image processing. Remote Sensing of Environment, 113, Supplement 1(0):S110 - S122, 2009.

[30] S.-E. Qian. Fast vector quantization algorithms based on nearest partition set search. IEEE Transactions on Image Processing, 15(8):24222430, 2006.

[31] P. Salembier and L. Garrido. Binary partition tree as an efficient representation for image processing, segmentation, and information retrieval. IEEE Transactions on Image Processing, 9(4):561-576, 2000.

[32] P. Soille. Morphological Image Analysis: Principles and Applications. Springer, 2nd edition, 2004.

[33] Y. Tarabalka, J. Chanussot, and J.A. Benediktsson. Segmentation and classification of hyperspectral images using minimum spanning forest grown from automatically selected markers. IEEE Transactions on Systems, Man, and Cybernetics, Part B: Cybernetics, 40(5):1267-1279, 2010.

[34] Y. Tarabalka, J. Chanussot, and J.A. Benediktsson. Segmentation and classification of hyperspectral images using watershed transformation. Pattern Recognition, 43(7):2367-2379, 2010.

[35] Y. Tarabalka, J.C. Tilton, J.A. Benediktsson, and J. Chanussot. A marker-based approach for the automated selection of a single segmentation from a hierarchical set of image segmentations. IEEE Journal of Selected Topics in Applied Earth Observations and Remote Sensing, 5(1):262-272, 2012.

[36] G. Tochon, J. Feret, R.E. Martin, R. Tupayachi, J. Chanussot, and G.P. Asner. Binary partition tree as a hyperspectral segmentation tool for tropical rainforests. In 2012 IEEE International Geoscience and Remote Sensing Symposium (IGARSS), pages 6368-6371, 2012.

[37] S. Valero. Hyperspectral image representation and Processing with Binary Partition Trees. phd, Grenoble Institute of Technology, 2011.

[38] S. Valero, P. Salembier, and J. Chanussot. Comparison of merging orders and pruning strategies for binary partition tree in hyperspectral data. In 2010 17th IEEE International Conference on Image Processing (ICIP), pages 2565-2568. IEEE, 2010.

[39] S. Valero, P. Salembier, and J. Chanussot. Hyperspectral image representation and processing with binary partition trees. IEEE Transactions on Image Processing, 22(4):1430-1443, 2013.

[40] M.A. Veganzones and M. Graña. Endmember extraction methods: A short review. In Knowledge-Based Intelligent Information and Engineering Systems, 12th International Conference, KES 2008, Zagreb, Croatia, September 3-5, 2008, Proceedings, Part III, volume 5179 of Lecture Notes in Computer Science, pages 400-407. Springer, 2008.

[41] M.A. Veganzones and M. Graña. A spectral/spatial cbir system for hyperspectral images. IEEE Journal of Selected Topics in Applied Earth Observations and Remote Sensing, 5(2):488-500, April 2012.

[42] M.A. Veganzones, G. Tochon, M. Dalla Mura, A. Plaza, and J. Chanussot. Hyperspectral image segmentation using a new spectral mixture-based binary partition tree representation. In 2013 20th IEEE International Conference on Image Processing (ICIP). IEEE, 2013.

[43] M.E. Winter. N-findr: an algorithm for fast autonomous spectral end-member determination in hyperspectral data. In Proceedings of SPIE, volume 3753, pages 266-275, 1999.

[44] P. Zhong and R. Wang. Learning conditional random fields for classification of hyperspectral images. IEEE Transactions on Image Processing, 19(7):1890-1907, 2010. 


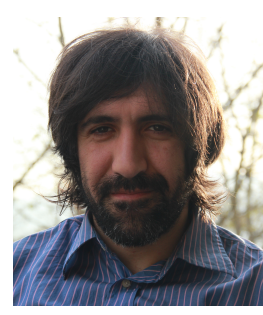

Miguel A. Veganzones (M'12) received the M.Sc. and Ph.D. degrees in computer science from the Basque Country University (UPV/EHU), Donostia, Spain, in 2005 and 2012 respectively. Since 2012, he is a posdoctoral researcher at the Images-Signal department in the GIPSA-lab, Grenoble, France. His current main research activities focus in the analysis of hyperspectral images by means of computational intelligence and statistical techniques. He is a Reviewer of IEEE Transactions on Geoscience and Remote Sensing, IEEE Geoscience and Remote Sensing Letters, IEEE Journal of Selected Topics in Earth Observations and Remote Sensing and the IEEE Journal of Selected Topics in Signal Processing.

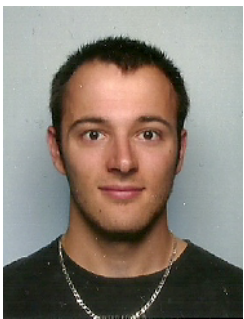

Guillaume Tochon received the M.Sc degree in Electrical Engineering from the Grenoble Institute of Technology (Grenoble-INP), France, in 2012, and is currently pursuing toward his Ph.D in signal and image processing at the University of Grenoble, with the Images-Signal department in the GIPSA-lab, France. He also serves as a reviewer for the IEEE Transactions on Geoscience and Remote Sensing and the IEEE Journal of Selected Topics in Earth Observations and Remote Sensing journals. He is interested in various domains of image processing, Graph theory and Information theory and their applications in remote sensing.

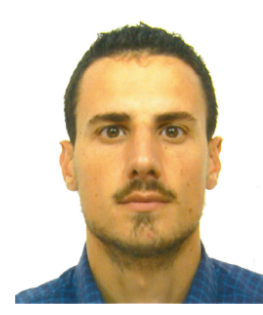

Mauro Dalla-Mura (S'08 - M'11) received the B.E. and M.E. degrees in Telecommunication Engineering from the University of Trento, Italy, in 2005 and 2007, respectively. He obtained in 2011 a joint Ph.D. degree in Information and Communication Technologies from the University of Trento, Italy and in Electrical and Computer Engineering from the University of Iceland, Iceland. He is currently an Assistant Professor at Grenoble Institute of Technology, France. $\mathrm{He}$ is conducting his research in the fields of remote sensing, image processing and pattern recognition at the Grenoble Images Speech Signals and Automatics Laboratory (GIPSA-Lab). He was the recipient of the IEEE GRSS Second Prize in the Student Paper Competition of the 2011 IEEE International Geoscience and Remote Sensing Symposium 2011. $\mathrm{He}$ is a member of the IEEE Geoscience and Remote Sensing Society (GRSS) and IEEE GRSS Data Fusion Technical Committee (DFTC). 


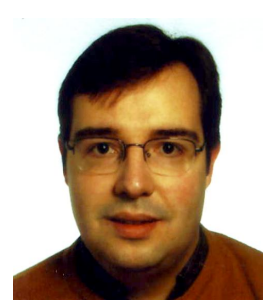

Antonio J. Plaza (M'05-SM'07) is an Associate Professor (with accreditation for Full Professor) with the Department of Technology of Computers and Communications, University of Extremadura, where he is the Head of the Hyperspectral Computing Laboratory (HyperComp). He was the Coordinator of the Hyperspectral Imaging Network, a European project with total funding of 2.8 MEuro (2007-2011). He authored more than 370 publications, including more than 100 JCR journal papers (60 in IEEE journals), 20 book chapters, and over 230 peer-reviewed conference proceeding papers (90 in IEEE conferences). He has guest edited seven special issues on JCR journals (three in IEEE journals). He has been a Chair for the IEEE Workshop on Hyperspectral Image and Signal Processing: Evolution in Remote Sensing (2011). He is a recipient of the recognition of Best Reviewers of the IEEE Geoscience and Remote Sensing Letters (in 2009) and a recipient of the recognition of Best Reviewers of the IEEE Transactions on Geoscience and Remote Sensing (in 2010), a journal for which he has served as Associate Editor in 2007-2012. He is also an Associate Editor for IEEE Access and the IEEE Geoscience and Remote Sensing Magazine, and was a member of the Editorial Board of the IEEE Geoscience and Remote Sensing Newsletter (2011-2012) and a member of the steering committee of the IEEE Journal of Selected Topics in Applied Earth Observations and Remote Sensing (2012). He served as the Director of Education Activities for the IEEE Geoscience and Remote Sensing Society (GRSS) in 2011-2012, and is currently serving as President of the Spanish Chapter of IEEE GRSS (since November 2012). He is currently serving as the Editor-in-Chief of the IEEE Transactions on Geoscience and Remote Sensing journal (since January 2013). Additional information: http://www.umbc.edu/rssipl/people/aplaza

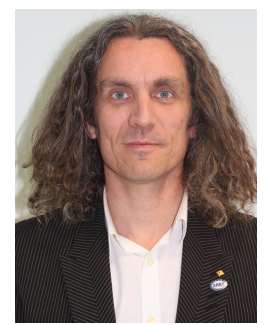

Jocelyn Chanussot (M04-SM04-F'12) received the M.Sc. degree in electrical engineering from the Grenoble Institute of Technology (Grenoble INP), Grenoble, France, in 1995, and the Ph.D. degree from Savoie University, Annecy, France, in 1998. In 1999, he was with the Geography Imagery Perception Laboratory for the Delegation Generale de l'Armement (DGA - French National Defense Department). Since 1999, he has been with Grenoble INP, where he was an Assistant Professor from 1999 to 2005, an Associate Professor from 2005 to 2007, and is currently a Professor of signal and image processing. He is conducting his research at the Grenoble Images Speech Signals and Automatics Laboratory (GIPSA-Lab). His research interests include image analysis, multicomponent image processing, nonlinear filtering, and data fusion in remote sensing. He is a member of the Institut Universitaire de France (2012-2017). Since 2013, he is an Adjunct Professor of the University of Iceland. Dr. Chanussot is the founding President of IEEE Geoscience and Remote Sensing French chapter (20072010) which received the 2010 IEEE GRS-S Chapter Excellence Award. He was the co-recipient of the NORSIG 2006 Best Student Paper Award, the IEEE GRSS 2011 Symposium Best Paper Award, the IEEE GRSS 2012 Transactions Prize Paper Award and the IEEE GRSS 2013 Highest Impact Paper Award. He was a member of the IEEE Geoscience and Remote Sensing Society AdCom (2009-2010), in charge of membership development. He was the General Chair of the first IEEE GRSS Workshop on Hyperspectral Image and Signal Processing, Evolution in Remote sensing (WHISPERS). He was the Chair (2009-2011) and Cochair of the GRS Data Fusion Technical Committee (2005-2008). He was a member of the Machine Learning for Signal Processing Technical Committee of the IEEE Signal Processing Society (2006-2008) and the Program Chair of the IEEE International Workshop on Machine Learning for Signal Processing, (2009). He was an Associate Editor for the IEEE Geoscience and Remote Sensing Letters (2005-2007) and for Pattern Recognition (2006-2008). Since 2007, he is an Associate Editor for the IEEE Transactions on Geoscience and Remote Sensing. Since 2011, he is the Editor-in-Chief of the IEEE Journal of Selected Topics in Applied Earth Observations and Remote Sensing. In 2013, he was a Guest Editor for the Proceedings of the IEEE and in 2014 a Guest Editor for the IEEE Signal Processing Magazine. 


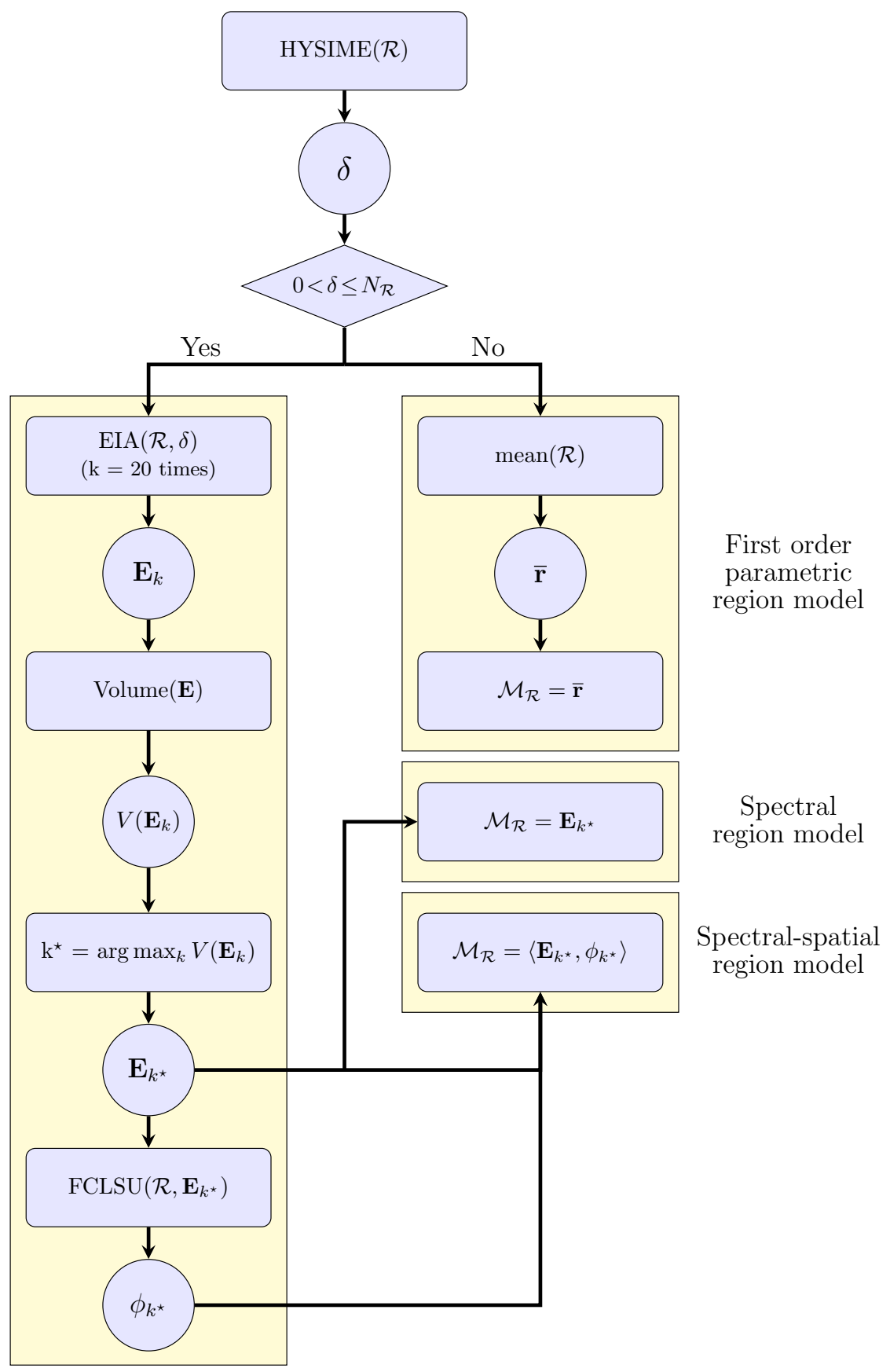

Unmixing Process

Fig. 4. Flow diagram of the spectral unmixing process to obtain the region model. 


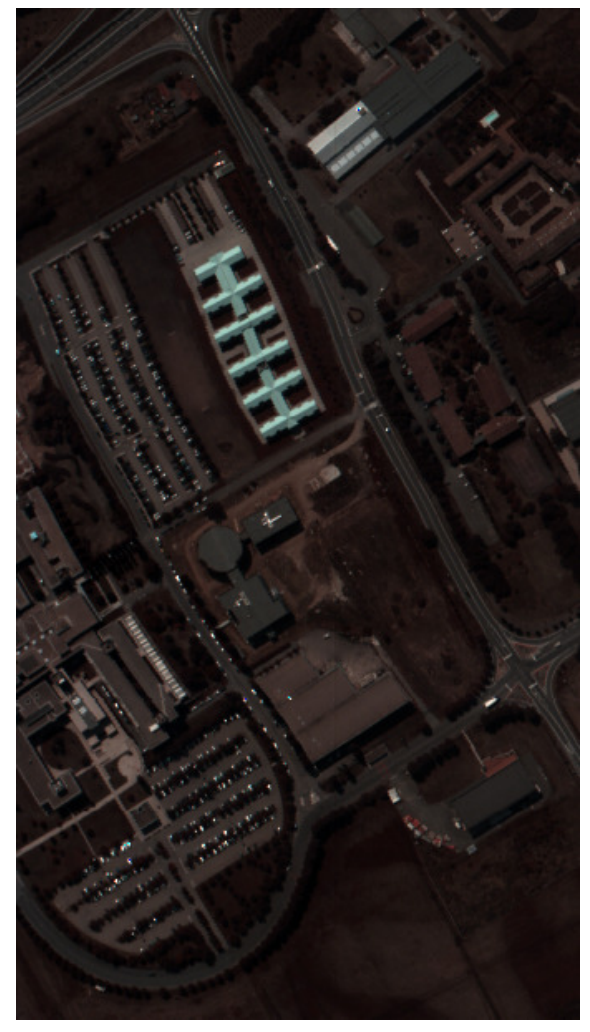

(a)

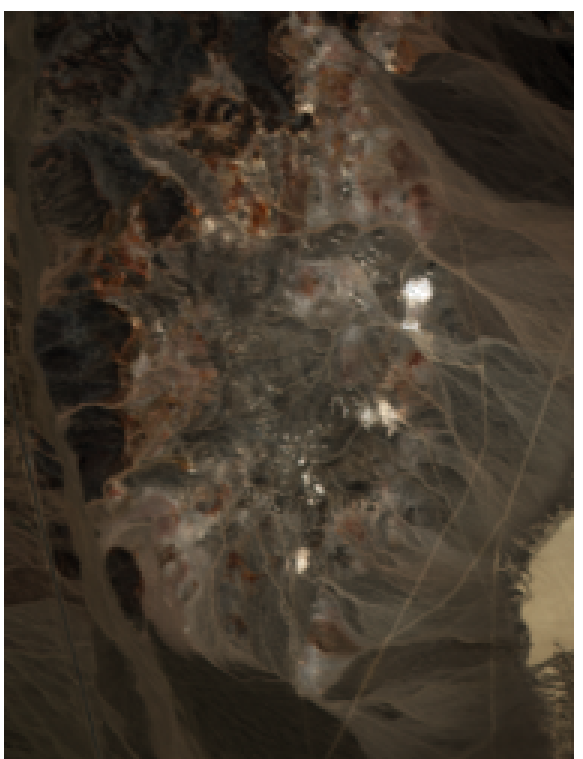

(b)

Fig. 5. (a) False color representation of the Pavia University scene and (b) The Cuprite scene. 

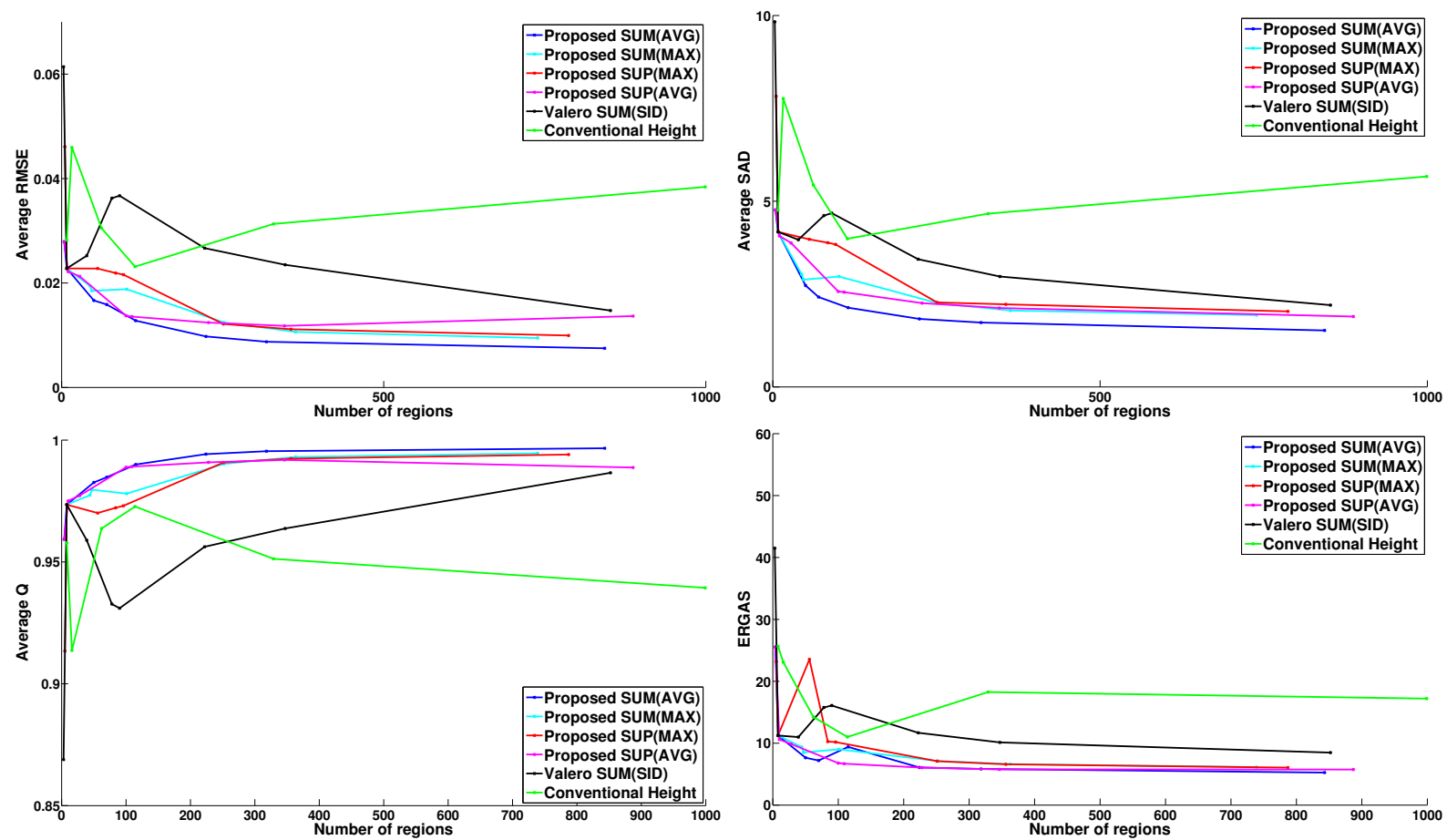

Fig. 6. Comparison of the different segmentation oriented pruning criteria in terms of unmixing reconstruction quality for the BPT representation of Pavia University image, built using the first-order parametric region model: (top-left) Average RMSE, (top-right) Average SAD, (bottom-left) Average Q (1 is the best), and (bottom-right) ERGAS. 

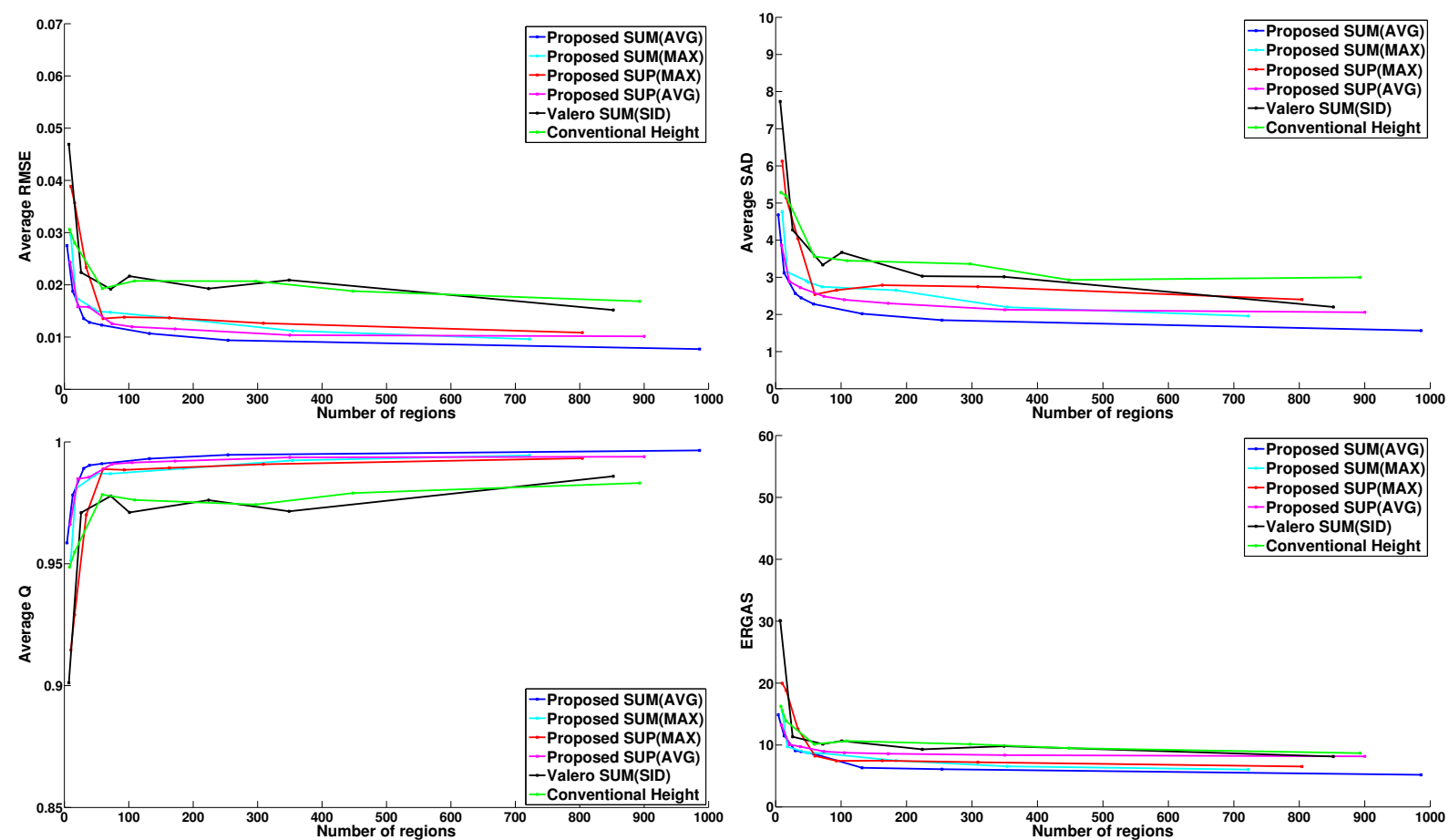

Fig. 7. Comparison of the different segmentation oriented pruning criteria in terms of unmixing reconstruction quality for the BPT representation of Pavia University image, built using the spectral region model: (top-left) Average RMSE, (top-right) Average SAD, (bottom-left) Average Q (1 is the best), and (bottom-right) ERGAS. 

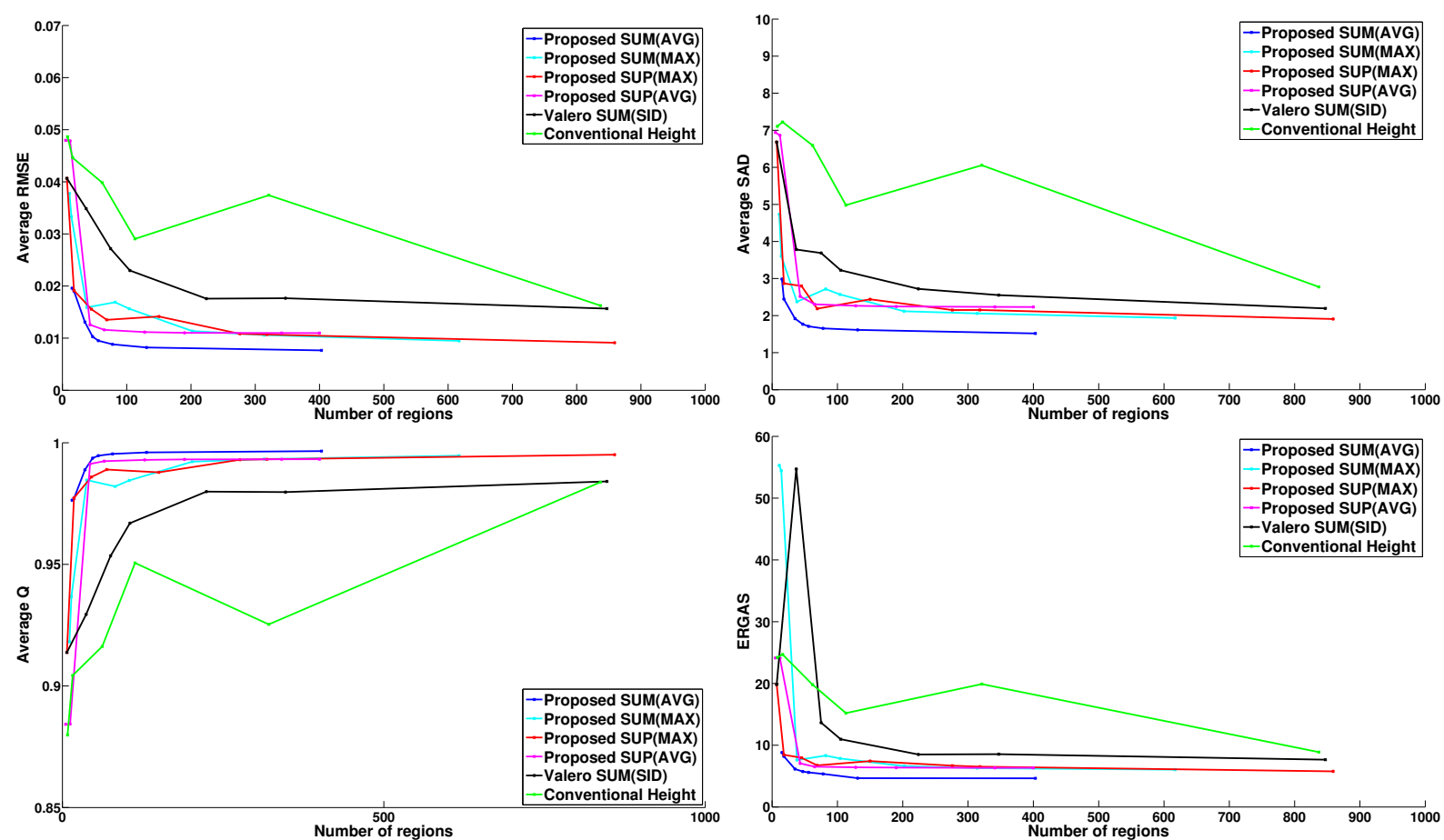

Fig. 8. Comparison of the different segmentation oriented pruning criteria in terms of unmixing reconstruction quality for the BPT representation of Pavia University image, built using the spectral-spatial parametric region model: (top-left) Average RMSE, (top-right) Average SAD, (bottomleft) Average Q (1 is the best), and (bottom-right) ERGAS. 


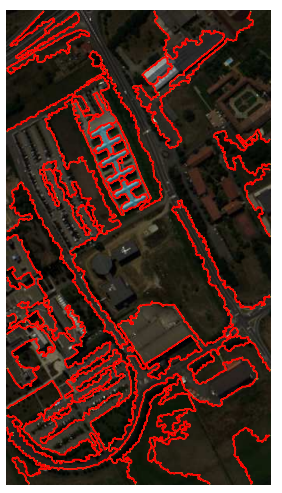

(a)

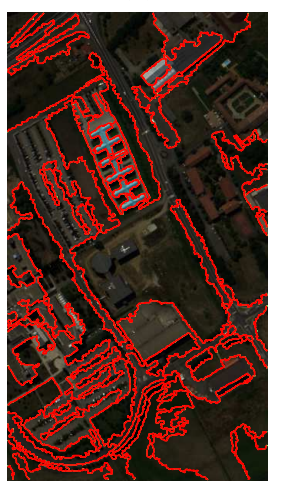

(f)

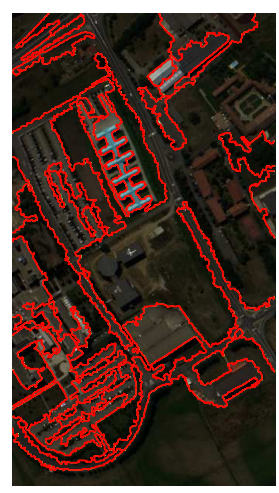

(b)

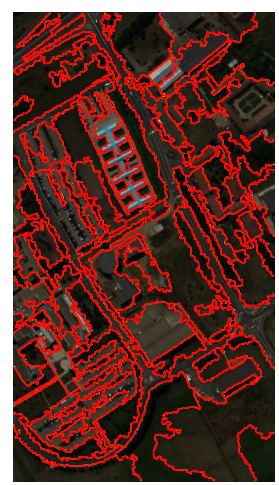

(g)

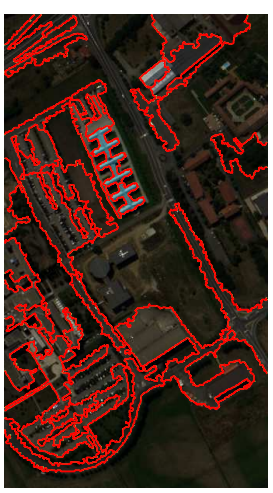

(c)

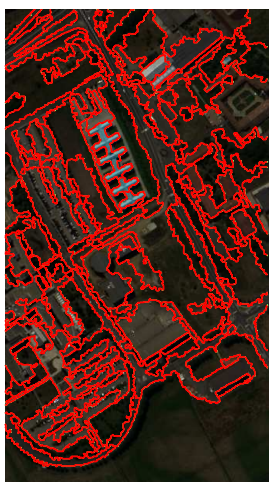

(h)

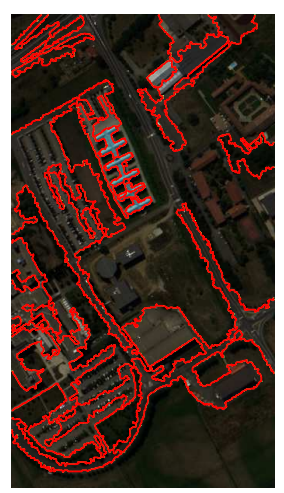

(d)

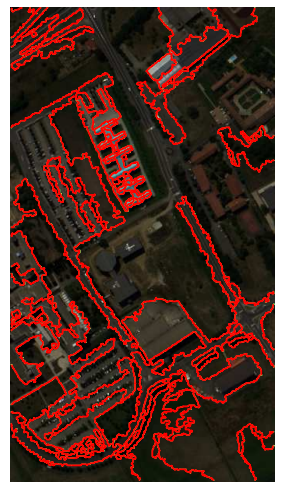

(i)

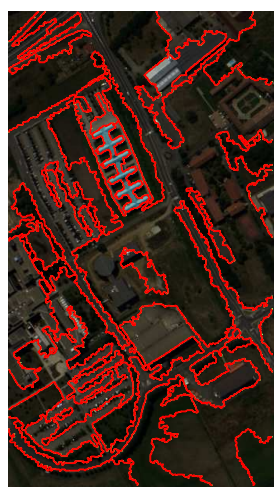

(e)

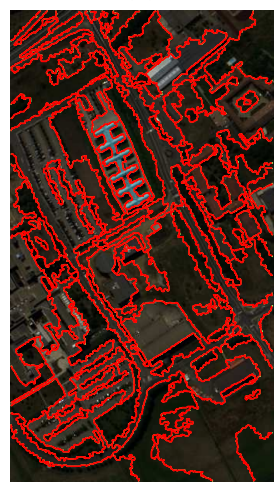

(j)

Fig. 9. Segmentations of Pavia University scene obtained by: (from left to right) Proposed SUM(AVG), Proposed SUM(MAX), Proposed SUP(MAX), Proposed SUP(AVG), and VALERO SUM(SID). Top row segmentations have around 50 regions, bottom row segmentations have around 100 regions. 

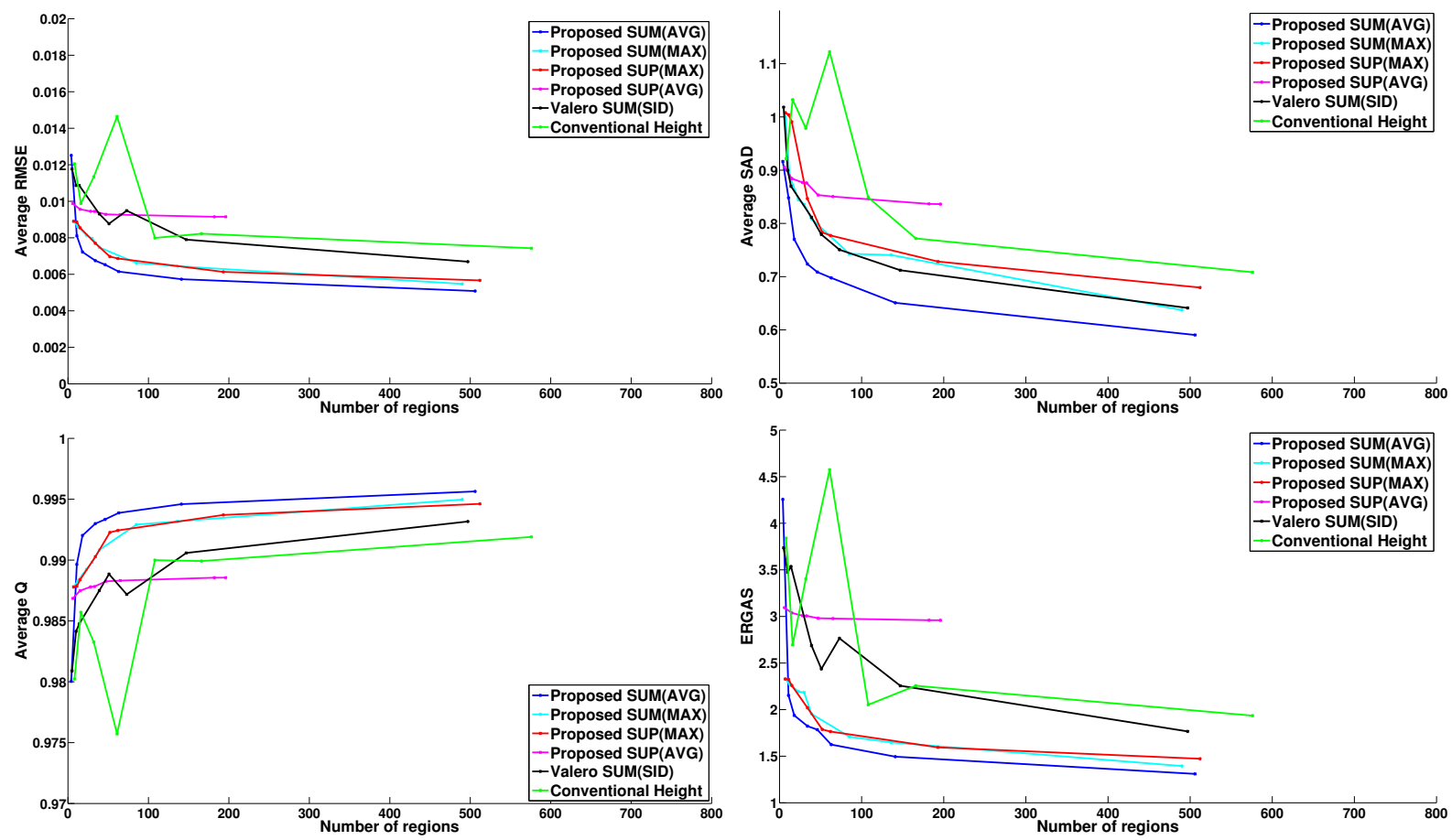

Fig. 10. Comparison of the different segmentation oriented pruning criteria in terms of unmixing reconstruction quality for the BPT representation of Cuprite image, built using the first-order parametric region model: (top-left) Average RMSE, (top-right) Average SAD, (bottom-left) Average Q (1 is the best), and (bottom-right) ERGAS. 

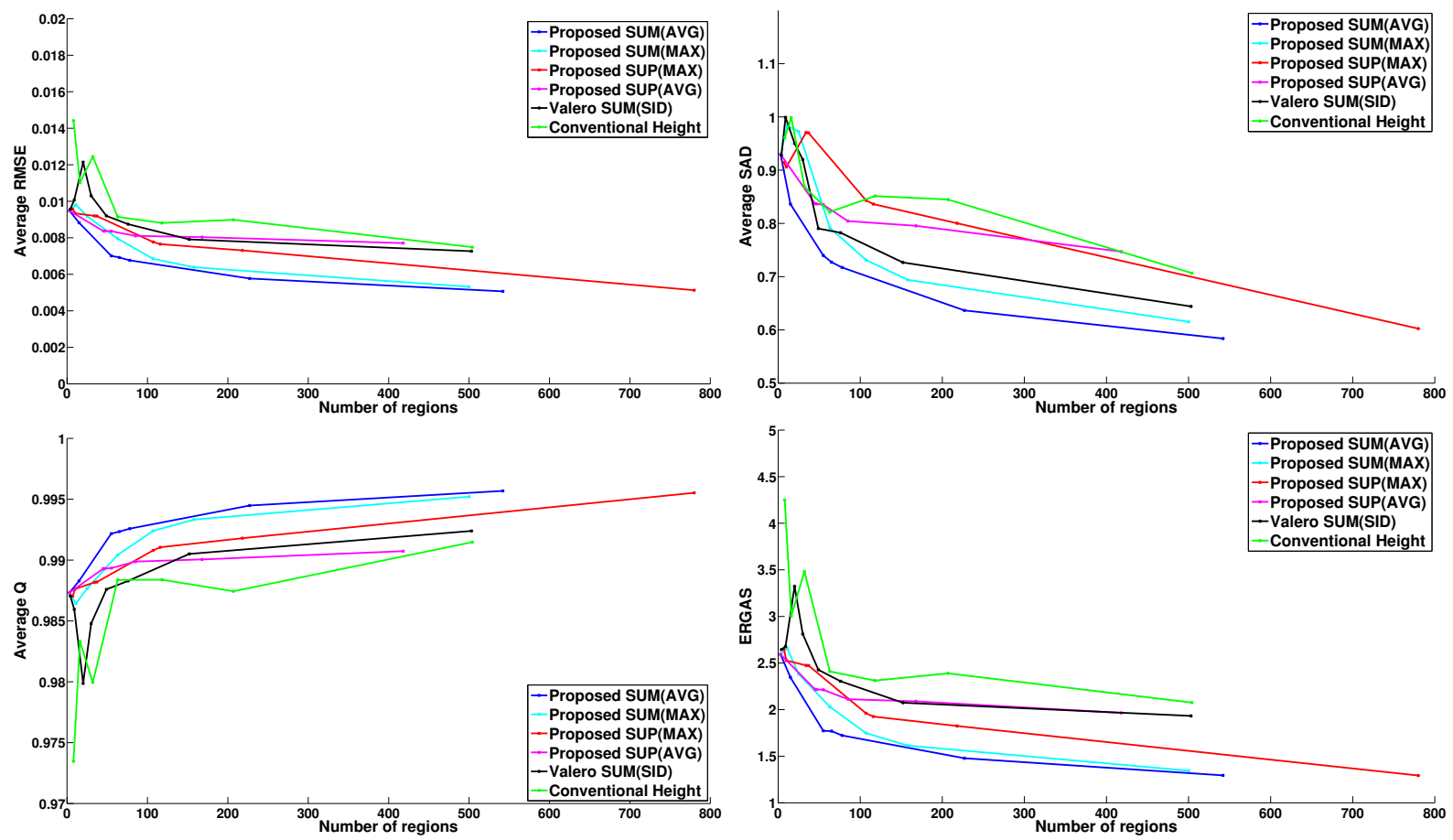

Fig. 11. Comparison of the different segmentation oriented pruning criteria in terms of unmixing reconstruction quality for the BPT representation of Cuprite image, built using the spectral region model: (top-left) Average RMSE, (top-right) Average SAD, (bottom-left) Average Q (1 is the best), and (bottom-right) ERGAS. 

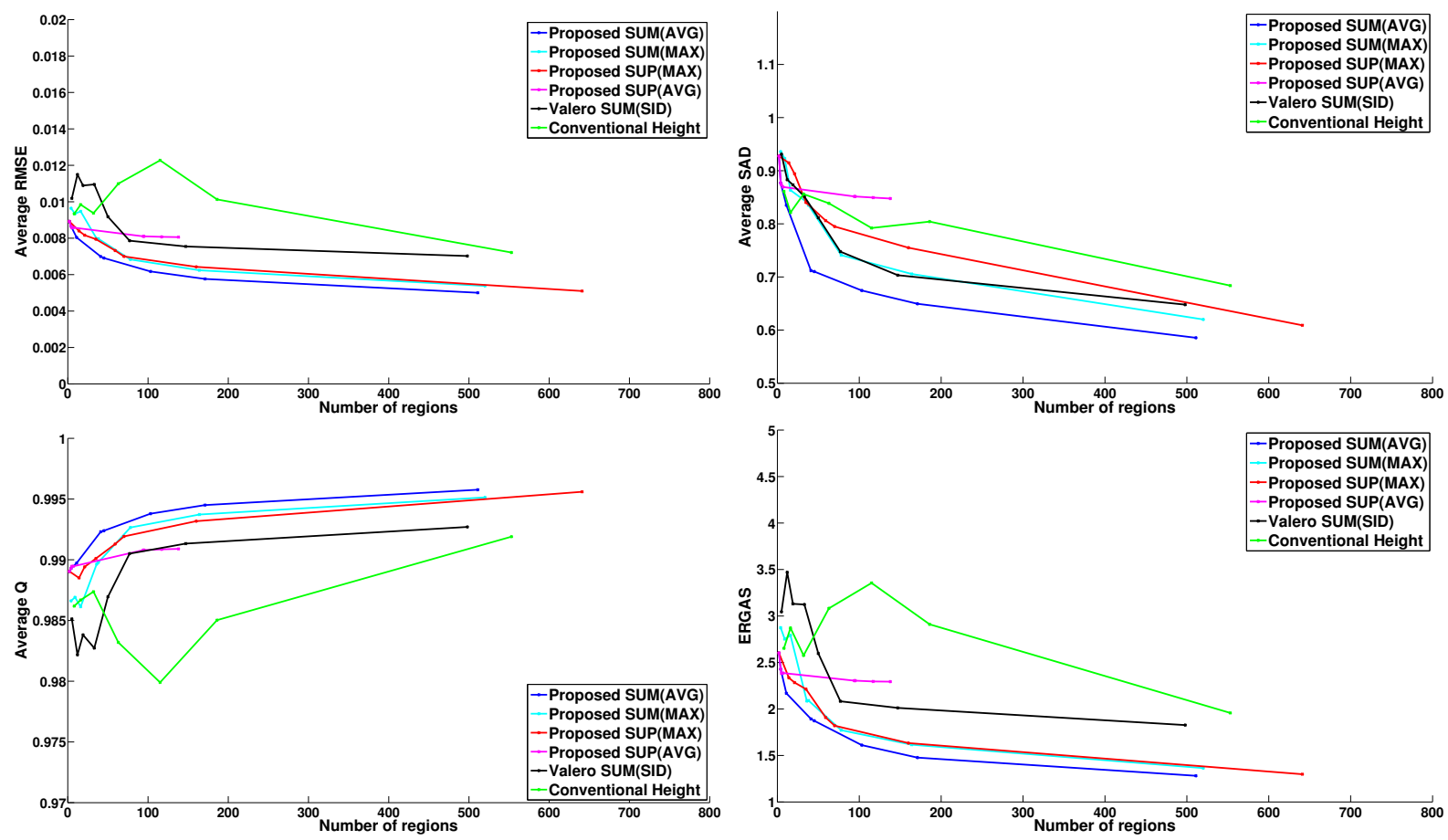

Fig. 12. Comparison of the different segmentation oriented pruning criteria in terms of unmixing reconstruction quality for the BPT representation of CUprite image, built using the spectral-spatial parametric region model: (top-left) Average RMSE, (top-right) Average SAD, (bottom-left) Average Q (1 is the best), and (bottom-right) ERGAS. 


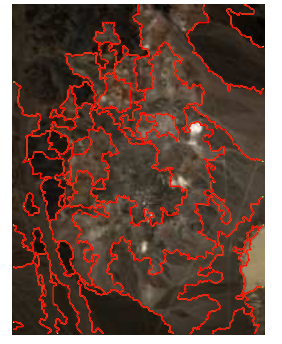

(a)

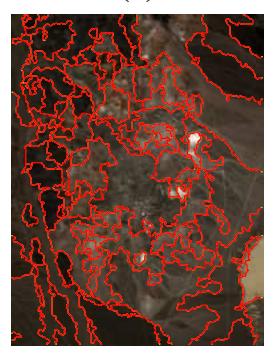

(f)

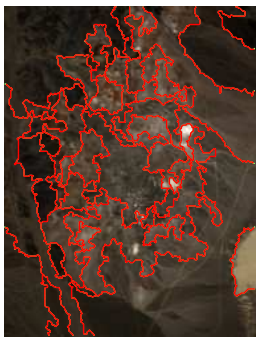

(b)

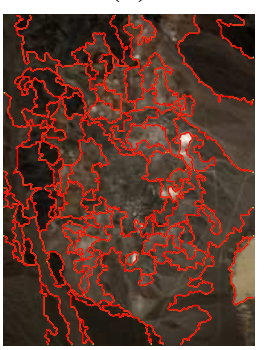

(g)

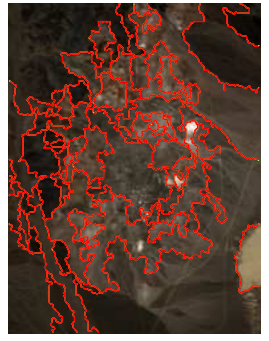

(c)

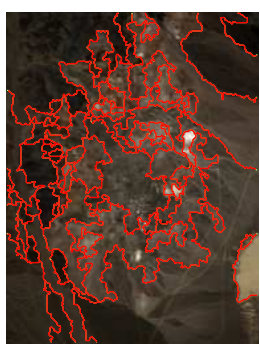

(h)

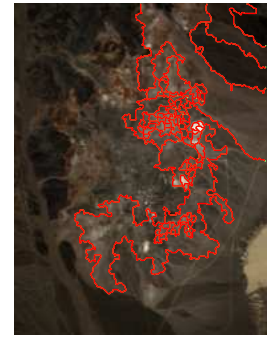

(d)

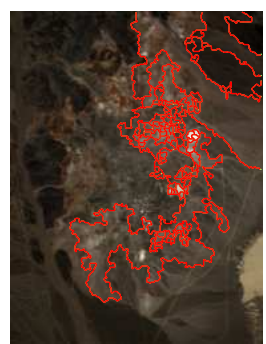

(i)

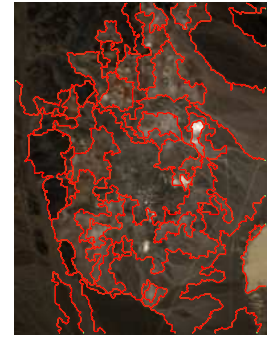

(e)

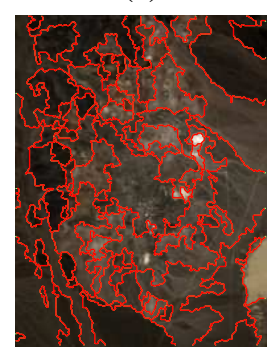

(j)

Fig. 13. Segmentations of Cuprite scene obtained by: (from left to right) Proposed SUM(AVG), Proposed SUM(MAX), Proposed SUP(MAX), Proposed SUP(AVG), and VALERO SUM(SID). Top row segmentations have around 50 regions, bottom row segmentations have around 100 regions. 